\title{
Distinct roles of core autophagy-related genes (ATGs) in zebrafish definitive hematopoiesis
}

\author{
Xiang-Ke Chen ${ }^{1}$, Zhen-Ni Yi ${ }^{1}$, Jack Jark-Yin Lau ${ }^{1}$, and Alvin Chun-Hang Ma ${ }^{1}$ \\ ${ }^{1}$ Department of Health Technology and Informatics, The Hong Kong Polytechnic University, Hong Kong, \\ China
}

\section{Address for Correspondence:}

Dr. Alvin Chun-Hang MA, PhD; Department of Health Technology and Informatics, Rm. Y924, Lee Shau

Kee Building, The Hong Kong Polytechnic University, Hung Hom, Hong Kong. Tel: +852 34008913; Fax:

+852 23624365; E-mail: alvin.ma@polyu.edu.hk 


\begin{abstract}
Despite the well-described discrepancy between some of the macroautophagy/autophagy-related genes (ATGs) in the regulation of hematopoiesis, the varying essentiality of core ATGs in vertebrate definitive hematopoiesis remains largely unclear. Here, we employed zebrafish (Danio rerio) to compare the function of six core atgs from the core autophagy machineries, which included $\operatorname{atg} 13$, beclin1 (becn1), $\operatorname{atg} 9 a, \operatorname{atg} 2 a, \operatorname{atg} 5$, and $\operatorname{atg} 3$, in vertebrate definitive hematopoiesis via CRISPR-Cas9 ribonucleoprotein targeting. Zebrafish embryos with various atg mutations showed autophagic deficiency throughout the body, including hematopoietic cells. The atgs mutations unsurprisingly caused distinctive hematopoietic abnormalities in zebrafish. Notably, becnl or atg9a mutation resulted in hematopoietic stem cells (HSCs) expansion during the development of the embryo into a larva, which can be attributed to the proteomic changes in metabolism, HSCs regulators, and apoptosis. Besides, atg3 mutation lowered the leukocytes in developing zebrafish embryos. Intriguingly, a synergistic effect on HSCs expansion was identified in atg $13+$ becn 1 and atg $9 a+a \operatorname{tg} 2 a$ or atg 3 double mutations, in which atg 13 mutation and $\operatorname{atg} 2 a$ or atg 3 mutation exacerbated and mitigated the HSCs expansion in becn 1 and atg $9 a$ mutations, respectively. In addition, the myeloid cell type-specific effects of various atgs were also determined between neutrophils and macrophages. Of these, a skewed ratio of neutrophils versus macrophages was found in atg 13 mutation, while both of them were reduced in atg3 mutation. These findings demonstrated the distinct roles of atgs and their interplays in zebrafish definitive hematopoiesis, thereby suggested that the vertebrate definitive hematopoiesis is regulated in an atgs-dependent manner.
\end{abstract}

Keywords: autophagy-related genes (ATGs); CRISPR-Cas9 ribonucleoprotein (RNP); definitive hematopoiesis; hematopoietic stem cells; zebrafish embryos 


\section{Abbreviations}

ATGs: autophagy-related genes; BECN1: Beclin1; CHT: caudal hematopoietic tissue; CKO: conditional knockout; CQ: Chloroquine; CRISPR: Clustered Regularly Interspaced Short Palindromic Repeats; dpf: days post fertilization; FIP 200: FAK family-interacting protein of $200 \mathrm{kDa}$; HSCs: hematopoietic stem cells; KD: knockdown; LC3: microtubule-associated protein 1A/1B-light chain 3; MO: Morpholino; PI3K: class 3 phosphoinositide 3-kinase; RFLP: restriction fragment length polymorphism; RNP: ribonucleoprotein; ULK: unc-51 like autophagy activating kinase; WISH: whole-mount in suit hybridization.

\section{Introduction}

Macroautophagy (hereinafter autophagy) serves as the scavenger of the cells by removal of harmful components via the lysosomal degradation, which, as an essential but complex process, is tightly regulated by a category of genes, namely autophagy-related genes (ATGs) [1]. Approximately 20 core ATGs that orchestrate the critical steps of canonical autophagy were classified into six functional groups/ machineries, including the unc-51 like autophagy activating kinase (ULK) 1 complex (initiation), class 3 phosphoinositide 3-kinase (PI3K) complex (isolation membrane nucleation), ATG9a vesicles (providing membrane), ATG2a complex (membrane elongation), ATG12 conjugation system (membrane elongation and linking microtubule-associated protein 1A/1B-light chain 3 [LC3] with PE), and LC3-PE conjugation system (membrane elongation and target recognition), which are highly conserved across the eukaryotes [2]. Loss of core ATGs commonly results in neonatal lethality in mice, while the exact cause of death remains elusive [3]. Despite the well-known essentiality of core ATGs in mice, a recent clinical study reported the identification of twelve patients from five families who survived with severely impaired autophagy due to the loss of ATG7, one of the most well studied core ATGs [4]. This was ascribed to the non-canonical or alternative autophagy 
that undergoes in the absence of some core ATGs since the autophagosome could still be formed [4,5]. Many core ATGs-independent autophagy, such as those that bypass ULK1, Beclin1 (BECN1), ATG5, and ATG7, as well as the autophagy-independent functions of core ATGs have been identified in the past decade [6,7]. Nevertheless, the majority of previous studies still focused on canonical autophagy, and the distinctive effects of various core ATGs are largely neglected.

Previous studies have shown the resemblance and discrepancy between core ATGs in the regulation of hematopoiesis, which is a vital biological process of blood cellular components formation, although very few of them included more than one ATG [8]. In definitive hematopoiesis, ATG5, ATG7, or FAK family-interacting protein of $200 \mathrm{kDa}$ (FIP200) conditional knockout (CKO) in hematopoietic cells declined the number of hematopoietic stem cells (HSCs) [9-11], whereas BECN1 and ATG12 CKO failed to alter the total number of HSCs [12,13]. Moreover, ATG5 or ATG7 CKO reduced the number of multi-lineage progenitors $[9,10]$; in contrast, their number increased and remained unchanged in ATG12 and BECN1 CKO, respectively [12,13]. Furthermore, ATG7, ATG12, or FIP200 CKO but not BECN1 as well as ATG5 CKO resulted in myeloproliferation, while ATG5, ATG7, or FIP200 CKO but not ATG12 or BECN1 CKO caused anemia [9-13]. These differential hematopoietic abnormalities found in these CKO of the core ATGs demonstrated the core ATGs-dependency of hematopoiesis, while more studies on other core ATGs are needed. In addition to the differences between ATGs, contradictory results were also observed in core ATGs CKO, including ATG5 and ATG7 [9,13,14], which indicate the important 'timing' of various core ATGs in the regulation of hematopoiesis. For instance, ATG7 is indispensable for adult but not neonatal HSCs in mice [14]. However, whether this 'timing' also occurs in other core ATGs or not remains unexplored. 
Over the past decades, zebrafish (Danio rerio), a tropical freshwater fish, has emerged as a vital genetic model in the field of hematopoiesis as well as autophagy due to its genetic tractability, small and transparent body, in vitro embryogenesis, and, more importantly, evolutionarily conversed genes orthologous to $70 \%$ of the human genes [15-17]. With the advanced gene editing tools, such as Clustered Regularly Interspaced Short Palindromic Repeats (CRISPR)-Cas9 ribonucleoprotein (RNP) complex [18], zebrafish embryos has gradually become a more efficient, convenient, and feasible model than mice in genetic screening in vivo, particularly in the study of hematopoiesis. Nevertheless, the roles of core atgs in zebrafish hematopoiesis remain largely unknown. Here, we reported for the first time, the differential effects of atgs mutations via CRISPR-Cas9 RNP targeting on zebrafish definitive hematopoiesis, although their mutations all disturbed the autophagy process, which highlighted the essentiality of various atgs-dependent effects rather than the uniformed canonical autophagy-dependent effect in vertebrate definitive hematopoiesis.

\section{Results}

\section{Core atgs targeting by CRISPR-Cas9 ribonucleoprotein (RNP)}

Core ATGs that are required for autophagy process have been classified in to six evolutionarily conserved functional groups, also known as 'core autophagy machineries' [2]. In this study, core atgs was selected form the six core machineries of autophagy for CRISPR-Cas9 sgRNP complex targeting, including atg13 (ULK1 complex), becn1 (PI3K complex), atg9a (ATG9a vesicles), atg2a (atg2a complex), atg5 (ATG12 conjugation system), and atg3 (LC3-PE conjugation system) (Figure 1A-B). Phylogenetic analysis revealed that these atgs were conserved between zebrafish and humans with high similarities (Figure S1A-D). crRNA were designed for each atg targeting early exon to model null-like mutation in zebrafish embryos (Figure 1C). Very high mutagenesis efficiencies ( $>95 \%$ ) were observed in all somatic atgs mutants as shown by restriction fragment 
length polymorphism (RFLP) assay (Figure 1F) and Sanger sequencing (Figure S1E), which can result in a null-like loss of atg protein (Figure S2A). In addition, a stable production of null-like zebrafish mutants can be achieved by the skilled operator (Figure S2B). While most of the mutant embryos (> 75\%) displayed normal development and morphology in embryonic stages (Figure 1D), deformed embryos could also been found (Figure 1E). However, most of these mutants, except $\operatorname{atg} 9 a$ and atg $2 a$ mutants can only survive up to around 2 week-post-fertilization.

\section{Autophagy deficiency in core atgs mutant zebrafish embryos}

We next examined autophagy after atgs targeting in $\mathrm{Tg}(\mathrm{Lc} 3$ :GFP) zebrafish line. The number of Lc3+ autophagy puncta (autophagosome) in the muscle was significantly lowered after $\operatorname{atg} 13$, becn1, atg 9 a, atg 5 , and atg 3 targeting but not in atg2a targeting (Figure 2A-C). The inconsistent autophagic activation or basal level was not only detected among various atgs mutations but also among various organs and tissues (Figure S2C). Intriguingly, all the atgs mutations cannot completely block autophagy, which suggested the presence of individual atg-independent alternative autophagy. In addition, autophagy flux was measured upon Chloroquine (CQ) treatment. An elevated number of puncta was observed in the control (CTRL), becnl, atg9a, and $\operatorname{atg} 5$ mutants after CQ treatment, while it remained unchanged in $\operatorname{atg} 13, \operatorname{atg} 2 a$, and $\operatorname{atg} 3$ mutants (Figure 2A-C). In particular, the number of puncta in atg $2 a$ mutants remain unchanged with or without CQ treatment, which suggested that its primary function in the blockage of autophagic flux. However, its mechanism is distinct from CQ, where the lysosome can still fuse with autophagosome but cannot undergo subsequent degradation (Figure 2D-G). Inconsistent with the observed autophagic changes in muscle cells, western blot of whole embryos showed a similar reduction in the Lc3II level in various atgs mutants other than atg 13 , which also indicated the tissue-specific autophagic changes (Figure 2H-I). A more reliable and sensitive 
CytoID staining [19] was performed in the sorted corola+ hematopoietic cells since very few puncta and Lc3 signal can be detected in the corola + cells of $\operatorname{Tg}(\mathrm{Lc} 3$ :GFP; corola:DsRed) zebrafish line (Figure S2D) and CytoID staining failed to penetrate the live embryo. Importantly, CytoID+ autophagic vacuoles declined in almost all atgs mutants similar to the decreased number of puncta in the muscle (Figure 3A-C), demonstrated that atgs mutations resulted in autophagy deficiency in the hematopoietic cells.

\section{Distinct effects of atgs mutation on definitive hematopoiesis}

We then examined the effects of atgs mutations on definitive hematopoiesis by whole-mount in suit hybridization (WISH). While all the atgs mutations resulted in autophagy deficiency, only atg 13, becnl, and atg3 mutations affected the number of $c-m y b+$ HSCs and $l c p l+$ pan-leukocytes in the caudal hematopoietic tissue (CHT), albeit modestly (<30\% of hematopoietic cells) (Figure 4A-C and 4G-I). More importantly, atg13 and atg3 mutations reduced but becnl mutation increased c-myb+ HSCs, while atg13 and becnl increased but atg3 decreased the lcpl+ pan-leukocytes. These differential hematopoietic phenotypes supported that the effects of atgs mutations on HSCs and leukocytes are possibly canonical autophagy-independent. In contrast, spil + myeloid progenitor declined in all the atgs mutants probably through the regulation of canonical autophagy, though more evidence is needed (Figure 4D-F). In addition, no significant difference was observed in the hbael+ erythrocytes in the CHT, which also suggested the hematopoietic linage-specific effect of atgs (Figure 4J-L). To elucidate the differential effects of atgs mutation in definitive hematopoiesis, mass spectrometry-based proteomic analysis was performed to identify the proteomic differences underlying the inconsistent phenotypes (Figure 5A). Strikingly, key proteins identified in proteomic analysis $(\mathrm{p}<0.05$, fold change $>1.50$ or $<0.66)$ varied between atgs mutations, in which beclin1 mutations have the largest number of altered proteins comparing with CTRL (Figure 5B-C). 
We further compared the proteomic profiles from different atgs mutants (Figure 5D-E and Figure S3). Interestingly, relatively more proteomic changes were observed in atg 13, beclin1, and atg5 mutants, while relatively less proteomic changes were found among atg $2 a$, atg 5 , and atg3 mutants. KEGG pathways enrichment suggested that metabolic pathways, biosynthesis pathways, and RNA and splicesome are the top three pathways that were changed in atgs mutations (Figure 5F). Among the atgs, less pathways were found in $\operatorname{atg} 3, \operatorname{atg} 2 a$, and atg 5 mutants compared to becn1, atg9a, and atg13. These pathways are potentially cooperated to influence definitive hematopoiesis under atgs mutants and autophagy deficiency. In particular, a number of HSC regulation-related proteins previously reported were also detected in the proteomic analysis, which were predominantly changed in becnl and atg9a mutants, while remained mostly unchanged in atg 5 and atg3 mutants (Figure 5G). Similarly, differential proteomic patterns can also be found in apoptosis-related proteins, which indicated an inhibition of apoptosis in zebrafish embryos under atgs mutations, especially under becnl mutation (Figure 5H). Therefore, the roles of atgs in definitive hematopoiesis could be, at least partially, attributed to specific proteins that modulate the programmed death, cell cycle, proliferation, and differentiation of hematopoiesis cells, which warrants further investigations.

\section{Time-dependent effects of atgs double mutations}

To further delineate the effects of atgs mutations on definitive hematopoiesis, we co-mutated core atgs selectively based on their differential proteomic profiles by co-injecting two sgRNPs targeting two atgs and high mutagenic efficiencies similar to single sgRNP injection were obtained (Figure 6A and Figure S2E). Since atgs has been found to be activated rhythmically in a clock-dependent manner, featuring the dynamic mRNA level of atgs during the days after birth [20], we also tracked the effect of atgs mutations on definitive hematopoiesis from 2 to $4 \mathrm{dpf}$ to examine the time-dependent effects of atgs in definitive hematopoiesis. 
Notably, becn1, atg13+becn1, and becn1+atg5 mutations resulted in aberrant accumulation of $c m y b+$ HSCs at $4 \mathrm{dpf}$, where a interaction effect was found in becn $1+\operatorname{atg} 13(\mathrm{~F}=4.27, p<0.05$, two-way ANOVA) but not in becn1+atg5 ( $p>0.05$, two-way ANOVA) (Figure 6B-C). Besides, atg 13 and $\operatorname{atg} 13+\operatorname{atg} 5$ mutations only declined the number of $c m y b+\mathrm{HSCs}$ at $2 \mathrm{dpf}$. While atg $9 a$ mutation also increased the $c m y b+\mathrm{HSCs}$ at $4 \mathrm{dpf}$, which can be alleviated by co-introduction of atg $2 a(\mathrm{~F}=18.02, p<0.01$, two-way ANOVA $)$ or atg3 $(\mathrm{F}=$ 16.25, $p<0.01$, two-way ANOVA) mutation (Figure 6D-E). Other single injections or co-injections showed no effects on $c m y b+$ HSCs. For coro1a+ leukocytes, although atg 13, becn1, atg 5, and atg $13+$ atg 5 mutations leaded to increased corola+ leukocytes, atg13+becnl showed an opposite effect, in which atg13+becnl attenuated the number of corola+ leukocytes (Figure 7A-C). These results indicated the different mechanisms underlying the increased corola+ leukocytes in becnl and atg 5 or atg13 mutants. While the increased corola + leukocytes in atg13, becnl, and atg 5 mutants were no longer observed on $4 \mathrm{dpf}$, both effects of atg $13+$ becn 1 and atg13+atg5 co-mutation on leukocytes lasted to 4 dpf though interaction effect was only observed in atg13+becnl $(\mathrm{F}=13.70, p<0.01$, two-way ANOVA) but not in $\operatorname{atg} 13+\operatorname{atg} 5(p>0.05$, two-way ANOVA) (Figure 7B-C). In addition, atg3, atg9a+atg $2 a, \operatorname{atg} 9 a+\operatorname{atg} 3$, and $\operatorname{atg} 2 a+\operatorname{atg} 3$ mutations resulted in the decreased corola+ leukocytes from 2 to $4 \mathrm{dpf}$. Main effect of $\operatorname{atg} 3(\mathrm{~F}=21.78, p<0.01$, two-way ANOVA) and $\operatorname{atg} 2 a(\mathrm{~F}=20.80, p<0.01$, two-way ANOVA) were observed in context of co-mutation with atg9a and no interaction effect ( $p>0.05$, two-way ANOVA) was found (Figure 7D-E). Altogether, these results indicate the distinctive molecular or cellular mechanism underlying different core atgs, which orchestrate in the regulation of definitive hematopoiesis.

\section{Effects of atgs mutation on myeloid lineages}

Comparison of atgs expression in various hematopoietic lineages was conducted in both human and zebrafish 
by in silico analysis of published gene expression database, which showed a huge variability and only a certain portion of hematopoietic cells expressed some of the ATGs/atgs (Figure S4A-B). Therefore, we further examined the effect of core atgs mutations on the two major types of myeloid cells, neutrophils and macrophages from 2 to $4 \mathrm{dpf}$ (Figure 8A). In accordance with the changes in corola+ leukocytes, atg 13 and becn 1 mutations elevated the number of $m p x+$ neutrophils at $2 \mathrm{dpf}$, while $\operatorname{atg} 9 a, \operatorname{atg} 2 a$, and $\operatorname{atg} 3$ showed the opposite effect (Figure 8B-C). In addition, atg 13, atg5, and atg3 mutations reduced the number of $m f a p 4+$ macrophages, while no significant difference was found in other atgs mutants (Figure 8D-E). Interestingly, although the number of corola+ leukocyte was not affected in atg13 mutant, a shift from mfap4+ macrophages to $m p x+$ neutrophils was observed (Figure 7B-C and Figure 8A-E). This indicated the opposite roles of atg 13 in the regulation of neutrophil and macrophage. Conversely, deceased corola+ leukocytes in atg3 mutant was resulted from both decreased $m p x+$ neutrophils and $m f a p 4+$ macrophages (Figure 7D-E and Figure 8A-E). Meanwhile, atgs mutation that only affected either neutrophil or macrophage population, such as the decreased neutrophils in $\operatorname{atg} 9 a$ or atg $2 a$ mutant and the decreased macrophages in $\operatorname{atg} 5$ mutant, were correlated to the subtle effects on total number of leukocytes (Figure 7 and Figure 8). Unlike $c m y b+$ HSCs and corola+ leukocytes, almost no time-dependent effect of atgs mutations on $m p x+$ neutrophils and $m f a p 4+$ macrophages was observed during the development form $2 \mathrm{dpf}$ to $4 \mathrm{dpf}$ in this study.

\section{Discussion}

Defective canonical autophagy has long been involved in the hematopoietic disturbances observed in core ATGs-deficient mice [21]. The evidence emerging in the past decade, however, reported the distinct effects of various core ATGs ablation on definitive hematopoiesis [9-13]. It suggested a canonical autophagy-independent role of core ATGs, which largely comprise core ATG-dependent non-canonical 
autophagy and non-autophagy functions, in the hematopoietic system, although very few core ATGs were compared. In this context, we hypothesized that the core ATG has both shared effects with other core ATGs and its own distinctive effects on definitive hematopoiesis. Therefore, we selected six core ATGs from different core autophagy machineries, including atg 13 (ulk1 complex), becn1 (PI3K complex), atg9a (atg9a vesicles), atg2a (atg2a complex), atg5 (atg12 conjugation system), and atg3 (Lc3-PE conjugation system) [2], and then examined their functions in definitive hematopoiesis by using CRISPR-Cas9 RNP targeting in the zebrafish model. We first observed the autophagy deficiency in the body and hematopoietic cells of various atgs-mutant zebrafish embryos while it varied among atgs. As expected, the autophagic and hematopoietic responses to atgs mutations were inconsistent. Mutation of core atgs has distinct effects on the definitive hematopoiesis, with some of the effects are time- or myeloid cell type-dependent. Furthermore, the interactions between core atgs in definitive hematopoiesis were also determined by double mutations, which revealed a synergistic effect between some of the core atgs; and demonstrated the interplays between atgs in zebrafish definitive hematopoiesis.

Approximately 20 core ATGs that are fundamental to canonical autophagy have been functionally categorized into six core autophagy machineries [2]. Since the canonical autophagy is essential for the development, mutations of core atgs corresponding to various core autophagy machineries except for $\operatorname{atg} 9 a$ and $a \operatorname{tg} 2 a$ in the present study resulted in the larval lethality in accordance with a previous report [22]. Notably, this indicated the similarity of Atgs/atgs in the early development between zebrafish and mice, while the exact cause of death needs further investigation [3]. As core ATGs that primarily facilitate the formation of autophagosome, autophagic responses to atg13, becn1, and atg5 knockout (KO) or knockdown (KD) have been previously studied in zebrafish, whereas studies on $\operatorname{atg} 9 a$, $\operatorname{atg} 2 a$, and $\operatorname{atg} 3$ mutations are not yet reported. Of these, 
morpholino (MO) KD of atg13 and atg5 decreased the level of autophagosome characterized by Lc3-II protein level or $L c 3+$ puncta in zebrafish [23,24], which are consistent with the reduced autophagosome observed in this study after CRISPR-Cas9 RNP complex-based atg 13 and atg5 targeting. Conversely, contradictory results were observed in becnl mutations, in which MO KD of becnl, exon two targeting in this study, and exon four targeting in the other study attenuated the level of autophagosome, while exon seven targeting in another study increased the level of autophagosome in zebrafish [24-26]. These results suggested a mutation type-dependent effect of becn 1 on autophagosome formation, and targeting an early exon of becnl may be required for the canonical autophagy deficiency. More importantly, to our knowledge, this is the first study that described autophagy defects in zebrafish embryos with atg $9 a$, atg $2 a$, and atg 3 mutations. Like $\operatorname{atg} 13$, becn1, and $\operatorname{atg} 5$, the functions of $\operatorname{atg} 9 a, \operatorname{atg} 2 a$, and $\operatorname{atg} 3$ in canonical autophagy of zebrafish are similar to their orthologs in mammalians. Because ATG9a and ATG3 are responsible for autophagosome formation and LC3 lipidation, respectively [27,28], a decreased level of autophagosome was detected in zebrafish with atg9a and atg3 mutations. In addition, loss of ATG2a is associated with impaired autophagic flux and accumulation of immature autophagosomal membranes in mammalian cells [29], which was reproduced in atg2a-mutant zebrafish embryos. Besides, atg13 and atg 3 mutations also affected the autophagic flux in zebrafish through a distinct mechanism by inhibiting the autophagosome formation and LC3 lipidation, respectively [28,30]. Altogether, the function of various atgs in the canonical autophagy process conservatively between zebrafish and mammalians, and CRISPR-Cas9 RNP targeting resulted in a similar loss of function as homozygous mutation and MO KD in zebrafish larvae.

Despite the autophagy deficiency was identified in zebrafish with various atgs mutations, our work showed that the hematopoietic alterations varied between atgs. The findings implied that their regulator effects are, at 
least partially, canonical autophagy-independent as observed in previous mice studies [9-13]. However, little is known about the specific core ATG-dependent hematopoietic effects in mice since none of them included a comparison between ATGs, and becnl and atg 5 are the only two core atgs out of six that were selected by this study that have been reported in previous mice studies. The present study determined that becnl mutation induced the expansion of HSCs during zebrafish embryonic development to larvae and transiently increased leukocytes or neutrophils. While the up-regulator effects of becn1 KD on HSCs has been reported in zebrafish with krill mutation [31], our work for the first time identified the sole role of zebrafish becnl in the expansion of HSCs in normal hematopoiesis, which can be ascribed to the directly regulated proteins of HSCs maintenance and disturbances among autophagy, apoptosis, and differentiation [32]. For instance, SWI/SNF related, matrix associated, actin dependent regulator of chromatin, subfamily c, member (smarcc) 1 a orthologous to human SMARCC1, which was previously shown as a positive regulator for zebrafish HSCs [33], was up-regulated following becn1 mutation. However, the level of HSCs, including long term-HSC and HSC-containing Lin-Sca-1+c-Kit+ cell, elevated in the spleen while declined in bone marrow in adult mice with Becnl CKO in hematopoietic cells [12]. Mouse HSCs reside in both spleen and bone marrow, whereas the HSCs largely resided in the CHT in zebrafish larvae. Therefore, whether Becn1/becnl behaves heterogeneously in zebrafish and mice and the precise roles of becn1 mutation requires the study on HSCs in the spleen and kidney marrow of adult zebrafish. Besides, this discrepancy between mice and zebrafish with Becn1/becn1 mutations can also be attributed to the unaltered autophagy and autophagic flux in HSCs of mice with Becnl CKO [12] and different developmental stages. Strikingly, myeloid cells-specific CKO of Becn1 increased the neutrophil and leukocytes but not the macrophages in mice, which was consistent with our findings in zebrafish, though the autophagic change in myeloid cells was not indicated [34]. Also, a similar expansion of hematopoietic lineages were reported in a fly model with atg6/becnl KO [35]. These findings 
indicated that the expansion of neutrophils and leukocytes in zebrafish with becnl mutation may be due to the loss of becnl in myeloid cells without affecting the HSCs. In addition to becnl mutation, atg9a mutation also caused HSCs expansion in zebrafish larvae. Although the study on ATG9a mutation in mammalian HSCs has not yet been reported, a similar proteomic response, especially in HSCs regulation and apoptosis between atg $9 a$ and becn 1 mutation, was presented in our study, which demonstrated a potential shared mechanism behind the atg9a and becnl in zebrafish HSCs regulation.

On the other hand, atg 5 mutation showed subtle effects on definitive hematopoiesis, HSCs in particular, in zebrafish larvae and also in mice model with ATG5 or ATG12 (core ATGs of ATG12 conjugation system) CKO, who also harbors a relatively healthy blood system [13]. Nevertheless, hematopoietic abnormalities were observed in mice with ATG5 CKO using a different hematopoietic promoter [9]. The dissimilarity between two mice studies could be explained by the vav:Cre CKO system (with abnormal blood system), which spontaneously effected much earlier than the Mx1:Cre CKO system, which needs the injection of 'triggers', such as PolyI:C in an older stage. Moreover, complete loss of atg5 induced $30 \%$ reduction of autophagosomes in the whole zebrafish embryo, whereas it led to an absence of autophagosomes in mouse neonates [36]. Taken together, atg5 plays a less important role in zebrafish larvae compared with mice; thus atg5 mutation was associated with a milder hematopoietic phenotype, which was also supported by the trend of change in the number of leukocytes and autophagic vacuoles in leukocytes with atg 5 mutation. In addition, mutation of atg3 that belongs to another conjugation system showed down-regulator effects on leukocytes, including both neutrophils and macrophages, which is in accordance with hematopoietic defects observed in ATG7-CKO mice probably due to the involvement of ATG7 in both ATG12 and LC3-PE conjugation systems [10]. Although the HSCs were reduced in adult mice with ATG7 CKO, recent work has revealed that ATG7 
CKO has no effect on the number and stemness of neonatal HSCs [14]. Similarly, HSCs remained unchanged in zebrafish larvae with either atg5 or atg3 mutation. Furthermore, both atg5 and atg 3 mutations attenuated the number of myeloid cells; in contrast, an expansion of myeloid cells accompanied with lymphopenia was commonly found in adult Atg7-CKO mice [10]. Thus, core atgs that belong to the core conjugation systems may act distinctly from the ATG7 in myeloid cells. This dissimilarity could also be ascribed to the varied developmental stages and mutations in different hematopoietic lineages as no adverse phenotype was manifested in mice with myeloid cell-specific ATG7 CKO [37]. Besides, a previous study also reported the cell-autonomous effect of FIP200, which together with ATG13 constitute the core autophagy machinery (ULK1 complex), CKO on mouse hematopoiesis. Loss of fetal HSCs and myeloid expansion were characterized in mice with FIP200 CKO [11]. Comparably, we found the atg13 mutation also elicited transiently decreased HSCs and increased leukocytes, as well as the skewing from macrophages to neutrophils. In summary, mutation of ATGs that belongs to the same autophagy machinery shared the feature of hematopoietic abnormalities, though more evidence is still needed.

In addition to the distinct effects of atgs on HSCs, leukocytes, and myeloid cells (neutrophils and macrophages), erythrocytes and myeloid progenitor cells showed similar responses to various atg mutations, which may be regulated through the canonical autophagy pathway. Specifically, myeloid progenitor cells were down-regulated, while erythrocytes remained constant. However, their responses in mice differed between ATGs CKO [9-13], which is potentially due to developmental stage difference [14]. More importantly, we determined the interactions between atgs, such as the synergistic effect of atg $13+$ becn 1 , atg $9 a+a \operatorname{tg} 2 a$, and atg $9 a+a \operatorname{tg} 3$ double mutations on HSCs expansion, which demonstrated the cooperation rather than sloe action of some of the ATGs in definitive hematopoiesis; and highlighted the essentiality of study with multiple ATGs. 
However, only one study with double ATGs KO was documented in mice, which revealed an indispensable Ulk1-mediated Atg5-independent autophagy in the regulation of erythropoiesis [38], while the interactions between other ATGs in other lineage hematopoiesis remain concealed. Despite our work providing a more comprehensive picture of various core ATGs in the regulation of definitive hematopoiesis than previous studies, the limitations of this study cannot be neglected: 1) the impact of single atg mutation is limited on zebrafish definitive hematopoiesis; 2) cell-autonomous and non-cell-autonomous effects of atgs cannot be distinguished, 3) the effect of atgs on adult hematopoiesis remains unclear, and 4) more ATGs and combinations are needed, which ultimately calls for the use of inducible hematopoietic cell-specific multiplex atgs $\mathrm{KO}$ in future studies.

\section{Materials and methods}

\section{Zebrafish strains and husbandry}

Transgenic and wild-type zebrafish were maintained in 14:10 h light:dark cycle and fed brine shrimp twice daily. $\operatorname{Tg}(\operatorname{Lc3}$ :GFP) [39], $\operatorname{Tg}$ (coro1a:DsRed) [40], $\operatorname{Tg}$ (cmyb:GFP), $\operatorname{Tg}$ (mpx:GFP), and $\operatorname{Tg}$ (mfap4:turquoise2) zebrafish lines were used in this study. $\operatorname{Tg}$ (mfap4:turquoise2) zebrafish line was generated by micro-injection of pDEST mfap4:turquoise2 plasmid construct (Addgene, 135218; David Tobin Lab) with in vitro transcribed tol2 transposase mRNA (Addgene, 31831; Stephen Ekker Lab). Zebrafish embryos were raised at $28.5^{\circ} \mathrm{C}$ and staged by day post $\square$ fertilization (dpf) and morphological criteria as previously described [41]. All animal experiments were performed in accordance with protocols approved by Animal Subjects Ethics Sub-Committee (ASESC) of The Hong Kong Polytechnic University.

\section{CRISPR-Cas9 sgRNP complex targeting}


Target sites of zebrafish atgs (atg13, becn1, atg9a, atg2a, atg5, and $\operatorname{atg} 3)$ and sgRNAs were identified and designed using Alt-R® CRISPR-Cas9 guide RNA design tool (Integrated DNA Technologies) and CRISPRscan [42]. Target sites were chosen that 1) a high on-target score, 2) a low off-target score, and 3) a restriction enzyme site around the protospacer adjacent motif (PAM) sequence were reported. Target sequence and restriction enzyme site of atgs sgRNA used in this study were listed in Figure 1C and Figure S1E. Given the target sequences of sgRNAs, CRISPR-Cas9 'single guide RNPs' (sgRNPs) was generated and delivered to zebrafish embryos as previously described [18]. Briefly, synthetic Alt-R® CRISPR-Cas9 sgRNA (Integrated DNA Technologies) was folded at $95^{\circ} \mathrm{C}$ for $5 \mathrm{~min}$ and then cooled down to the room temperature. Alt-R® S.p. Cas9 Nuclease V3 (Integrated DNA Technologies, 1081059) diluted in Cas9 working buffer (20 mM HEPES, $150 \mathrm{mM} \mathrm{KCl}, \mathrm{pH} 7.5$ ) and subsequently was assembled with folded sgRNA in $37^{\circ} \mathrm{C}$ for 10 min. Around $2 \mathrm{nl}$ sgRNP complex was delivered into the cell of zebrafish embryos at one-cell stage through microinjection. For co-injection, two sgRNPs was formed individually and then mixed before micro-injection. Around 4nl sgRNP complexes were injected into the cell of zebrafish embryos. Almost no toxic effect of these sgRNP complexes was observed in zebrafish embryos after microinjection.

\section{Detection of mutagenesis}

Mutagenesis and mutagenic efficiency were detected by using RFLP assay as previously described [43]. Briefly, a 300-600bp PCR fragment covering the designed sgRNA target site was amplified for each atgs using corresponding primers listed in Table S1. Next, restriction enzyme (listed in Figure S1E, New England Biolabs) was then used to cleave the PCR fragment, in which uncleaved band suggested a destroyed restriction enzyme site by sgRNP-induced mutations, while the cleaved band contained wild-type sequence. Mutagenic efficiency was calculated by dividing the intensity of uncleaved band with the intensity of total 
band measured by ImageJ (NIH). To further confirm the mutations, uncleaved band was sub-cloned into pGEM®-T Easy Vector (Promega Corporation, A1360) and the insertions and deletions (indels) were identified by Sanger sequencing.

\section{Western blotting}

The protein level of Lc3 and GAPDH in zebrafish embryos was measured using western blot as previously described [39]. Briefly, total protein extracted from dechorionated and deyolked embryos was resolved on 12\% gels (Bio-Rad Laboratories, 1610175) and then transferred to the PVDF membrane (Merck Millipore, IPVH00010). After blocking with 5\% nonfat dried milk (Bio-Rad Laboratories, 1706404), membrane was probed with anti-Lc3b (Abcam, ab48394) and anti-GAPDH (Cell Signaling Technology, 2118) primary antibodies. Afterward, the membrane was probed with goat anti-rabbit IgG secondary antibody (Invitrogen, 32460) and visualized using SuperSignal ${ }^{\mathrm{TM}}$ West Femto Maximum Sensitivity Substrate (Thermo Fisher, 34095) after wash with with Tris-buffered saline (50 mM Tris base, $150 \mathrm{mM} \mathrm{NaCl}, \mathrm{pH}$ 7.5) plus Tween-20 (Bio-Rad Laboratories, 1610781).

\section{LysoTracker Red and Cyto-ID staining}

Live zebrafish embryos was incubated with LysoTracker Red DND-99 (Invitrogen, L7528) at $10 \mu \mathrm{M}$ in E3 medium ( $5 \mathrm{mM} \mathrm{NaCl}, 0.17 \mathrm{mM} \mathrm{KCL}, 0.33 \mathrm{mM} \mathrm{CaCl}$, and $0.33 \mathrm{mM} \mathrm{MgSO} 4, \mathrm{pH}$ 7.4) for $30-45 \mathrm{~min}$ at $28.5^{\circ} \mathrm{C}$ in dark [39]. On the other hand, autophagic vacuoles and nucleus in sorted cells were immediately stained using CYTO-ID® Autophagy detection kit 2.0 with Hoechst 33342 Nuclear Stain (Enzo Life Sciences, ENZ-KIT175) at $37^{\circ} \mathrm{C}$ in dark following the manufacturer's instructions [19]. Wash with E3 medium and $1 \mathrm{X}$ assay buffer were applied to both LysoTracker Red and Cyto-ID live staining, respectively, before fluorescent 
microscopic imaging.

\section{Fluorescence-activated cell sorting}

Single-cell suspension for FACS was prepared as described previously [44]. $\operatorname{Tg}$ (coro1a:DsRed) zebrafish embryos at $3 \mathrm{dpf}$ were digested with Gibco ${ }^{\mathrm{TM}}$ Trypsin-EDTA (0.05\%) (Thermo Fisher, 25300062) for 15 minutes at $28^{\circ} \mathrm{C}$ and then disassociated with pipetting on ice. After termination of Trypsin with $\mathrm{CaCl} 2(2 \mathrm{mM})$, the suspension was filtered using $40 \mu \mathrm{m}$ cell strainer (BD Biosciences, 352340) and washed with phosphate-buffered saline (PBS) (VWR Life Science, E404-200TABS) with 1\% (vol/vol) fetal bovine serum (FBS) (Thermo Fisher, 26140079). FACS of corola:DsRed+ leukocytes was then conducted in BD FACSAria III Cell Sorter according to the manufacturer's instructions. Around 8,000 corola:DsRed + cells was targeted to be sorted using a purity sort mode.

\section{Fluorescent microscope imaging}

Fluorescent images of transgenic zebrafish embryos with or without LysoTracker Red staining or sorted corola + cells stained with Cyto-ID and Hoechst were taken by using Zeiss Lightsheet Z.1 Selective Plane Illumination Microscope, Leica TCS SPE Confocal Microscope, or Nikon Stereomicroscope with a Nikon DS-Fi2 Camera as previously described [39]. Zebrafish embryos were mounted in 1.5\% low-melting agarose (Sigma-Aldrich, A9045) into $35 \mathrm{~mm}$ glass-bottom confocal dish or glass capillary before imaging. Tricaine (Sigma-Aldrich, A5040) at $0.16 \mathrm{mg} / \mathrm{ml}$ in E3 medium was used as anaesthetic for zebrafish embryos. In addition, $\operatorname{Tg}(\mathrm{Lc} 3$ :GFP) zebrafish embryo was treated with chloroquine (Selleckchem, S4157) at $100 \mu \mathrm{M}$ in E3 medium before imaging. 


\section{Whole-mount in situ hybridization}

Whole-mount in situ hybridization (WISH) was performed on zebrafish embryos following the standard protocol described previously [44]. DIG-labeled anti-sense probes (cmyb, spil, lcpl, and hbael) were made from the pGEM®-T Easy vector (Promega Corporation, A1360) containing the gene-coding sequences via in vitro transcription using DIG RNA Labeling Kit (Roche, 11175025910). A short bleaching was used to remove the pigments from the fixed zebrafish embryos.

\section{Mass spectrometry-based proteomics}

Total protein was extracted from thirty zebrafish embryos using cell lysis buffer (Sigma-Aldrich, C3228). Purified protein was then digested into peptides using Trypsin (Promega, V5111) and desalted using Pierce C18 Spin Columns (Thermo Fisher, 89870). A label-free quantitative proteomics was conducted on Thermo Fisher Orbitrap Fusion Lumos Mass Spectrometer coupled with Dionex UltiMate 3000 RSLCnano. Identification and quantification was processed with Progenesis QI software, and the abundance of proteins was quantified based on three independent experiments and normalized based on total protein. Arbitrary fold change cut-offs of $>1.5$ and significance p-values of 0.05 were set for significantly up-regulated or down-regulated proteins.

\section{Quantification and statistics}

The number of Lc3+ puncta in the muscle was counted in Zeiss ZEN software following the criteria and protocol described in our previous study [39]. Cyto-ID+ autophagic vacuoles was defined by vacuole-like

GFP+ fluorescence signals that were distinguished from the background and a similar size of autophagic vacuoles was considered in all the autophagic vacuoles counting in the cells. In addition, ImageJ (NIH) was 
utilized to measure the relative intensity of proteins in western blot and straighten the WISH images. Data are reported as mean \pm standard deviation (S.D.). One-way ANOVA, two-way ANOVA, $C^{2}$ test, and independent t-test were performed where appropriate using Statistical Package for the Social Sciences (SPSS) Version 14.0 and a $p$-value less than 0.05 was considered statistically significant.

\section{Acknowledgements}

The zebrafish maintenance was supported by Fish Model Translational Research Laboratory (HTI, PolyU). Light-sheet and confocal fluorescent microscopic imaging, flow cytometry, and mass spectrometry-based proteomics were supported by University Research Facility in Life Sciences (ULS, PolyU).

\section{Disclosure statement}

No potential conflicts of interest were disclosed.

\section{References}

[1] Jiang P, Mizushima N. Autophagy and human diseases. Cell Res. 2014;24:69-79.

[2] Suzuki H, Osawa T, Fujioka Y, et al. Structural biology of the core autophagy machinery. Curr Opin Struct Biol. 2017;43:10-17.

[3] Kuma A, Komatsu M, Mizushima N. Autophagy-monitoring and autophagy-deficient mice. Autophagy. 2017;13:1619-1628.

[4] Collier JJ, Guissart C, Oláhová M, et al. Developmental Consequences of Defective ATG7-Mediated Autophagy in Humans. N Engl J Med. 2021;384:2406-2417.

[5] Nishida Y, Arakawa S, Fujitani K, et al. Discovery of Atg5/Atg7-independent alternative 
macroautophagy. Nature. 2009;461:654-658.

[6] Codogno P, Mehrpour M, Proikas-Cezanne T. Canonical and non-canonical autophagy: variations on a common theme of self-eating? Nat Rev Mol Cell Biol. 2011;13:7-12.

[7] Galluzzi L, Green DR. Autophagy-Independent Functions of the Autophagy Machinery. Cell. 2019;177:1682-1699.

[8] Sawyers CL, Denny CT, Witte ON. Leukemia and the disruption of normal hematopoiesis. Cell. $1991 ; 64: 337-350$.

[9] Jung HE, Shim YR, Oh JE, et al. The autophagy Protein Atg5 Plays a Crucial Role in the Maintenance and Reconstitution Ability of Hematopoietic Stem Cells. Immune Netw. 2019;19:e12.

[10] Mortensen M, Soilleux EJ, Djordjevic G, et al. The autophagy protein Atg7 is essential for hematopoietic stem cell maintenance. J Exp Med. 2011;208:455-467.

[11] Liu F, Lee JY, Wei H, et al. FIP200 is required for the cell-autonomous maintenance of fetal hematopoietic stem cells. Blood. 2010;116:4806-4814.

[12] Yang X, Ge L, Wang J. BECN1 modulates hematopoietic stem cells by targeting Caspase-3-GSDME-mediated pyroptosis. Blood Sci. 2020;2:89-99.

[13] Ho TT, Warr MR, Adelman ER, et al. Autophagy maintains the metabolism and function of young and old stem cells. Nature. 2017;543:205-210.

[14] Hashimoto M, Umemoto T, Nakamura-Ishizu A, et al. Autophagy is dispensable for the maintenance of hematopoietic stem cells in neonates. Blood Adv. 2021;5:1594-1604.

[15] Davidson AJ, Zon LI. The “definitive” (and 'primitive') guide to zebrafish hematopoiesis. Oncogene. $2004 ; 23: 7233-7246$.

[16] He C, Bartholomew CR, Zhou W, et al. Assaying autophagic activity in transgenic GFP-Lc3 and 
GFP-Gabarap zebrafish embryos. Autophagy. 2009;5:520-526.

[17] Howe K, Clark MD, Torroja CF, et al. The zebrafish reference genome sequence and its relationship to the human genome. Nature. 2013;496:498-503.

[18] Hoshijima K, Jurynec MJ, Klatt Shaw D, et al. Highly Efficient CRISPR-Cas9-Based Methods for Generating Deletion Mutations and F0 Embryos that Lack Gene Function in Zebrafish. Dev Cell. 2019;51:645-657.e4.

[19] Guo S, Liang Y, Murphy SF, et al. A rapid and high content assay that measures cyto-ID-stained autophagic compartments and estimates autophagy flux with potential clinical applications. Autophagy. 2015;11:560-572.

[20] Huang G, Zhang F, Ye Q, et al. The circadian clock regulates autophagy directly through the nuclear hormone receptor Nr1d1/Rev-erb $\alpha$ and indirectly via $\mathrm{Cebpb} /(\mathrm{C} / \mathrm{ebp} \beta)$ in zebrafish. Autophagy. 2016;12:1292-1309.

[21] Mortensen M, Watson AS, Simon AK. Lack of autophagy in the hematopoietic system leads to loss of hematopoietic stem cell function and dysregulated myeloid proliferation. Autophagy. 2011;7:1069-1070.

[22] Morishita H, Kanda Y, Kaizuka T, et al. Autophagy Is Required for Maturation of Surfactant-Containing Lamellar Bodies in the Lung and Swim Bladder. Cell Rep. 2020;33:108477.

[23] Masud S, Prajsnar TK, Torraca V, et al. Macrophages target Salmonella by Lc3-associated phagocytosis in a systemic infection model. Autophagy. 2019;15:796-812.

[24] Lee E, Koo Y, Ng A, et al. Autophagy is essential for cardiac morphogenesis during vertebrate development. Autophagy. 2014;10:572-587.

[25] Mawed SA, Zhang J, Ren F, et al. atg7 and beclin1 are essential for energy metabolism and survival 
during the larval-to-juvenile transition stage of zebrafish. Aquac Fish. 2021;

[26] Dong G, Zhang Z, Duan K, et al. Beclin 1 deficiency causes hepatic cell apoptosis via endoplasmic reticulum stress in zebrafish larvae. FEBS Lett. 2020;594:1155-1165.

[27] Imai K, Hao F, Fujita N, et al. Atg9A trafficking through the recycling endosomes is required for autophagosome formation. J Cell Sci. 2016;129:3781-3791.

[28] Nath S, Dancourt J, Shteyn V, et al. Lipidation of the LC3/GABARAP family of autophagy proteins relies on a membrane-curvature-sensing domain in Atg3. Nat Cell Biol. 2014;16:415-424.

[29] Tang Z, Takahashi Y, Chen C, et al. Atg2A/B deficiency switches cytoprotective autophagy to non-canonical caspase-8 activation and apoptosis. Cell Death Differ. 2017;24:2127-2138.

[30] Puente C, Hendrickson RC, Jiang X. Nutrient-regulated Phosphorylation of ATG13 Inhibits Starvation-induced Autophagy. J Biol Chem. 2016;291:6026-6035.

[31] Jia X-E, Ma K, Xu T, et al. Mutation of krill causes definitive hematopoiesis failure via PERK-dependent ～excessive autophagy induction. Cell Res. 2015;25:946-962.

[32] Wang J. Beclin 1 bridges autophagy, apoptosis and differentiation. Autophagy. 2008;4:947-948.

[33] Huang H-T, Kathrein KL, Barton A, et al. A network of epigenetic regulators guides developmental haematopoiesis in vivo. Nat Cell Biol. 2013;15:1516-1525.

[34] Tan P, He L, Xing C, et al. Myeloid loss of Beclin 1 promotes PD-L1hi precursor B cell lymphoma development. J Clin Invest. 2019;129:5261-5277.

[35] Shravage B V, Hill JH, Powers CM, et al. Atg6 is required for multiple vesicle trafficking pathways and hematopoiesis in Drosophila. Development. 2013;140:1321-1329.

[36] Kuma A, Hatano M, Matsui M, et al. The role of autophagy during the early neonatal starvation period. Nature. 2004;432:1032-1036. 
[37] Cao Y, Zhang S, Yuan N, et al. Hierarchal Autophagic Divergence of Hematopoietic System. J Biol Chem. 2015;290:23050-23063.

[38] Honda S, Arakawa S, Nishida Y, et al. Ulk1-mediated Atg5-independent macroautophagy mediates elimination of mitochondria from embryonic reticulocytes. Nat Commun. 2014;5:4004.

[39] Chen XK, Kwan JS, Chang RC, et al. 1-phenyl 2-thiourea (PTU) activates autophagy in zebrafish embryos. Autophagy. 2020/04/15. 2020;1-10.

[40] Yan B, Han P, Pan L, et al. IL-1 $\beta$ and reactive oxygen species differentially regulate neutrophil directional migration and Basal random motility in a zebrafish injury-induced inflammation model. J Immunol. 2014/05/20. 2014;192:5998-6008.

[41] Kimmel CB, Ballard WW, Kimmel SR, et al. Stages of embryonic development of the zebrafish. Dev Dyn. 1995/07/01. 1995;203:253-310.

[42] Moreno-Mateos MA, Vejnar CE, Beaudoin J-D, et al. CRISPRscan: designing highly efficient sgRNAs for CRISPR-Cas9 targeting in vivo. Nat Methods. 2015;12:982-988.

[43] Bedell VM, Wang Y, Campbell JM, et al. In vivo genome editing using a high-efficiency TALEN system. Nature. 2012;491:114-118.

[44] Ma AC, Ward AC, Liang R, et al. The role of jak2a in zebrafish hematopoiesis. Blood. 2007/06/05. 2007;110:1824-1830.

\section{Figure legends}

Figure 1. Core atgs targeting by CRISPR-Cas9 ribonucleoprotein (RNP). (A) Schematic diagram showing the involvement of autophagy machineries in autophagy pathway. PAS, pre-autophagosomal structure. (B) Schematic diagram showing the CRISPR-Cas9 RNP targeting and core autophagy-related genes (atgs) 
selected from autophagy machinery (cam). (C) Target sequences and sgRNA design of various atgs. (D) Morphology and percentage of normal morphology of zebrafish with atgs mutations. Scale bar, $0.5 \mathrm{~mm}$. (E) Representative deformed zebrafish embryos with atg mutants. Scale bar, $0.5 \mathrm{~mm}$. (F) Restriction fragment length polymorphism (RFLP) assay and mutation efficiency.

Figure 2. Autophagic deficiency in zebrafish embryos with core atgs mutations. (A-C) Autophagosomes or Lc3+ puncta in the muscle of $\operatorname{Tg}(\operatorname{Lc} 3: G F P)$ zebrafish embryos with (+) and without (-) Chloroquine (CQ) treatment in various atgs mutation. *, p $<0.05$ compared with CTRL or CQ-. **, p $<0.01$ compared with CTRL or CQ-. Scale bar, $50 \mu \mathrm{m}$. (D-G) Fusion of autolysosome between Lc3+ autophagosome and LysoTracker+ lysosome in $\operatorname{Tg}(\mathrm{Lc} 3: \mathrm{GFP})$ zebrafish embryos with (+) and without (-) CQ treatment in various atgs mutation. Scale bar, $5 \mu \mathrm{m}$. (H-I) Western blotting result of $L c 3$ and gapdh protein levels in zebrafish embryos with various atgs mutation.*, $\mathrm{p}<0.05$ compared with CTRL.

Figure 3. Attenuation of autophagic vacuoles in leukocytes with core atgs mutations. (A) Experimental setup for Cyto-ID+ autophagic vacuoles measurement in corola+ leukocytes. (B-C) Representative images and quantification of Cyto-ID+ autophagic vacuoles in corola+ leukocytes sorted from zebrafish embryos with various atgs mutation. *, p < 0.05 compared with CTRL. **, $\mathrm{p}<0.01$ compared with CTRL. Scale bar, $5 \mu \mathrm{m}$.

Figure 4. Distinct effects of core atgs mutation on definitive hematopoiesis in zebrafish. (A-C) Whole mount in situ hybridization (WISH) results of $c m y b+$ HSCs. ${ }^{* *}, \mathrm{p}<0.01$ compared with CTRL. (D-F) WISH results of spilb+ myeloid progenitor. ${ }^{*}, \mathrm{p}<0.05$ compared with CTRL. ${ }^{* *}, \mathrm{p}<0.01$ compared with CTRL. (G-I) WISH results of $l c p l+$ pan-leukocytes. **, p < 0.01 compared with CTRL. (J-K) WISH results of hbael+ 
erythrocytes. No significant difference was found between CTRL and atgs mutations $\left(C^{2}\right.$ test). All caudal hematopoietic tissue (CHT) from WISH pictures was straightened by ImageJ.

Figure 5. Mass spectrometry-based proteomic variability among atgs mutations. (A) Experimental setup for mass spectrometry-based proteomic analysis of zebrafish embryos with various atgs mutations. (B-C) Volcanic map and heat map of comparison between atgs mutation and CTRL. Red dots, p $<0.05$ and fold change > 1.5. (D-E) Comparison of the number (\#) of changed proteins, increased proteins, decreased proteins, and the same protein among atgs mutation. (F-H) Comparison of KEGG pathways and specific proteins (HSCs and apoptosis-related) among atgs mutation.

Figure 6. Time-dependent effects of core atgs single or double mutations on HSCs. (A) Experimental setup for the time-dependent responses of $c m y b+$ HSCs to single or double mutations of atgs. (B-C) Effect of combination among atg 13, becn1, and atg 5 mutations on $c m y b+$ HSCs during the period from $2 \mathrm{dpf}$ to $4 \mathrm{dpf}$. *, $\mathrm{p}<0.05$ compared with CTRL. **, $\mathrm{p}<0.01$ compared with CTRL. \#\#, $\mathrm{p}<0.01$ compared with becn1 mutation. (D-E). Effect of combination among atg $9 a$, atg $2 a$, and atg 3 mutations on $c m y b+$ HSCs during the period from $2 \mathrm{dpf}$ to $4 \mathrm{dpf}$. *, p < 0.05 compared with CTRL.

Figure 7. Time-dependent effects of core atgs single or double mutations on leukocytes. (A) Experimental setup for the time-dependent responses of corola+ leukocytes to single or double mutations of atgs. (B-C) Effect of combination among atg13, becn1, and atg 5 mutations on corola + leukocytes during the period from 2 dpf to 4 dpf. *, p < 0.05 compared with CTRL. **, p $<0.01$ compared with CTRL. \#\#, p $<0.01$ compared with becnl mutation. (D-E). Effect of combination among atg9a, atg $2 a$, and atg 3 mutations on corola+ 
leukocytes during the period from $2 \mathrm{dpf}$ to $4 \mathrm{dpf}$. *, p $<0.05$ compared with CTRL. **, p $<0.01$ compared with CTRL.

Figure 8. The effect of core atgs mutation in myeloid lineages. (A) Experimental setup for the time-dependent responses of $m p x+$ neutrophils and mfap4+ macrophages to atgs mutation. (B-C) Effect of atg13, becnl, $\operatorname{atg} 9 a, \operatorname{atg} 2 a, \operatorname{atg} 5$, and $\operatorname{atg} 3$ mutations on $m p x+$ neutrophils during the period from 2 dpf to 4 dpf. *, p $<0.05$ compared with CTRL. **, p < 0.01 compared with CTRL. (D-E) Effect of atg13, becnl, atg9a, atg2a, atg5, and atg 3 mutations on mfap $4+$ macrophages during the period from 2 dpf to 4 dpf. *, p $<0.05$ compared with CTRL. **, $\mathrm{p}<0.01$ compared with CTRL. 
$A$

ULK1 complex

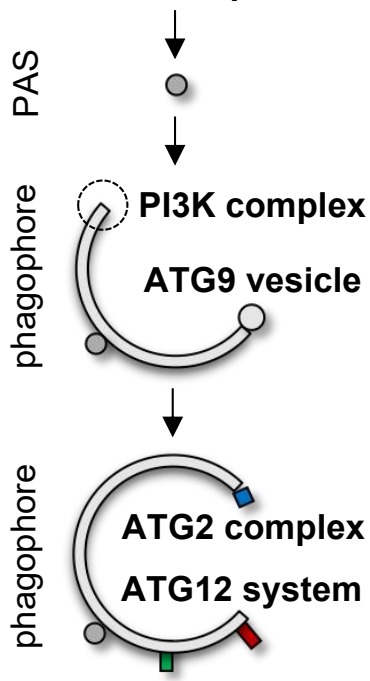

LC3-PE system

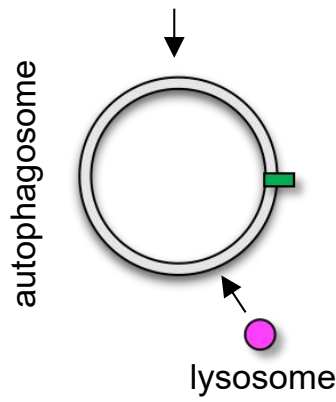

$\mathrm{F}$

B

mancouna

gene
(1) $\operatorname{atg} 13$
2 becn1
3 atg9a
$4 \operatorname{atg}^{2} a$
5 atg5
6 atg3
atgs

C

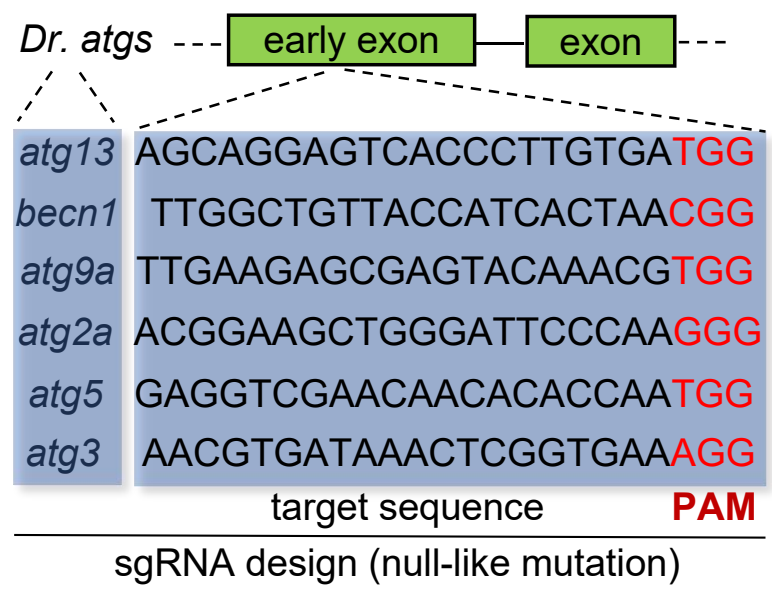

ULK1 complex PI3K complex ATG9 vesicle ATG2 complex ATG12 system

LC3-PE system machineries

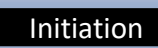

Nucleation

Nucleation

Elongation

Elongation

Elongation

roles
D
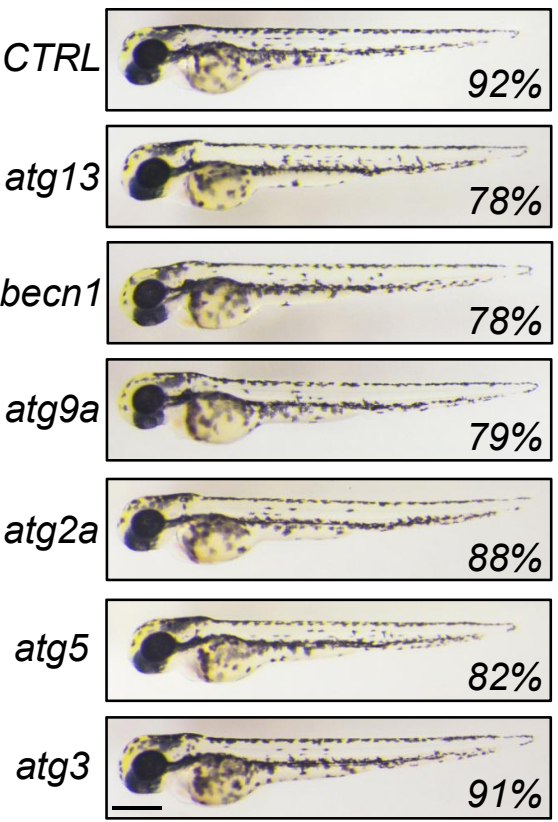

$E$
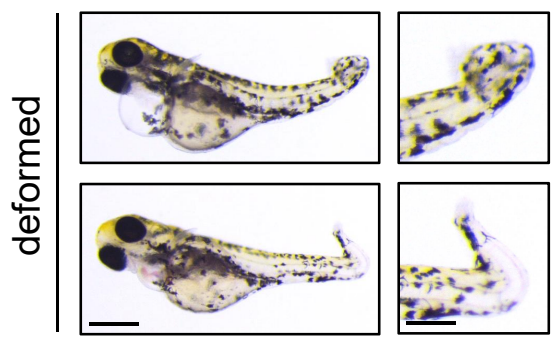
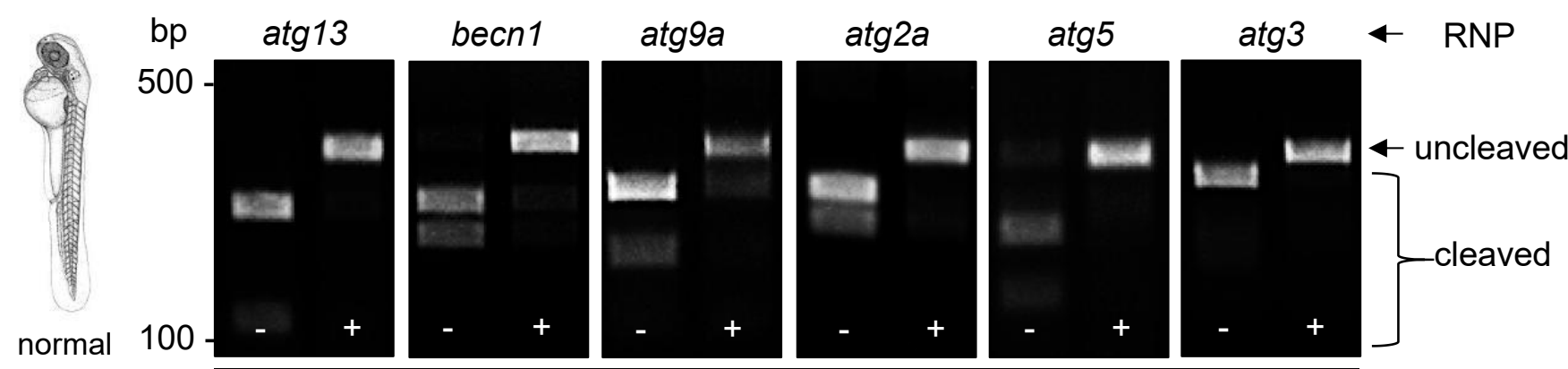

morphology

RFLP assay (mutagenesis efficiency $>95 \%$ ) 


$$
\text { leukocyte } \rightarrow \text { autophagy }
$$

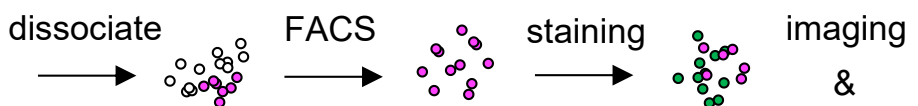
filter corola $\quad$ CytolD ${ }^{\infty}$ counting

coro1a

C
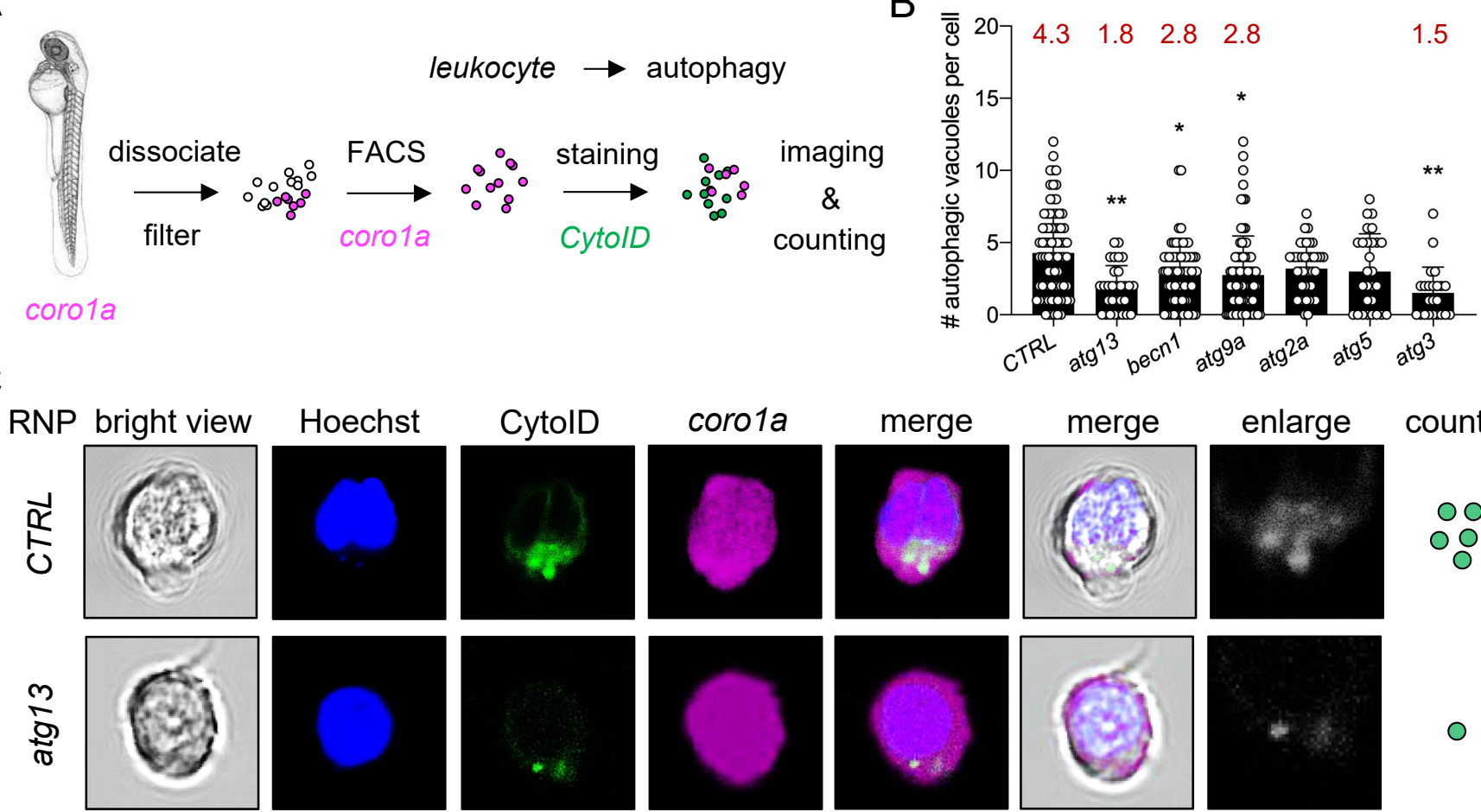

counting
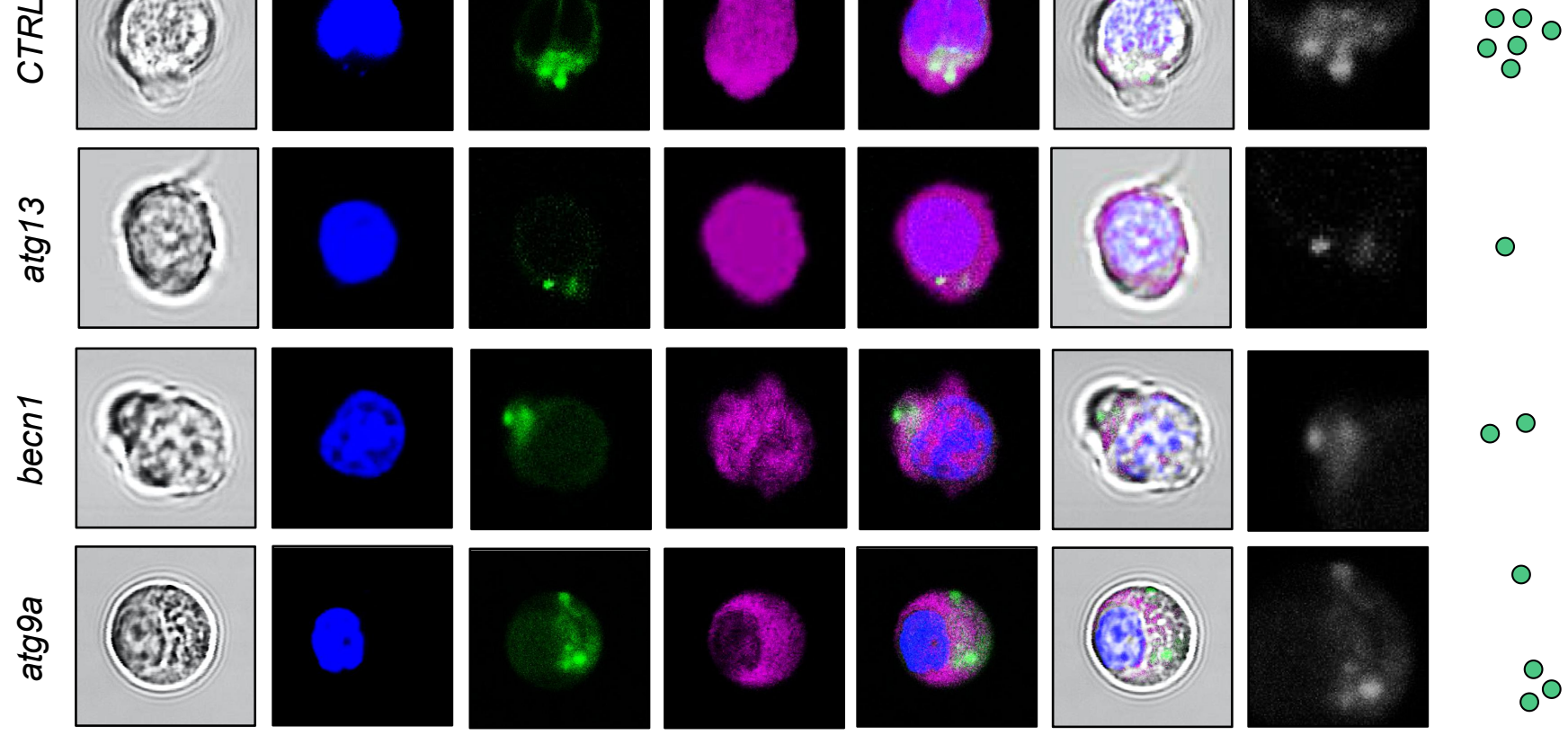

$\circ \bigcirc$
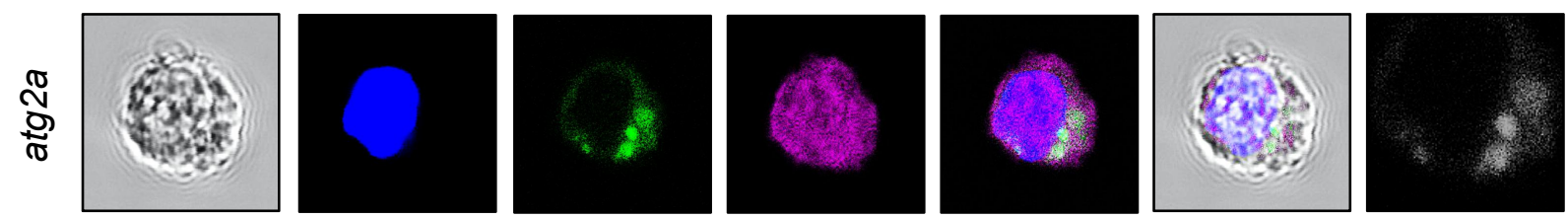

$\mathrm{O}^{\circ}$
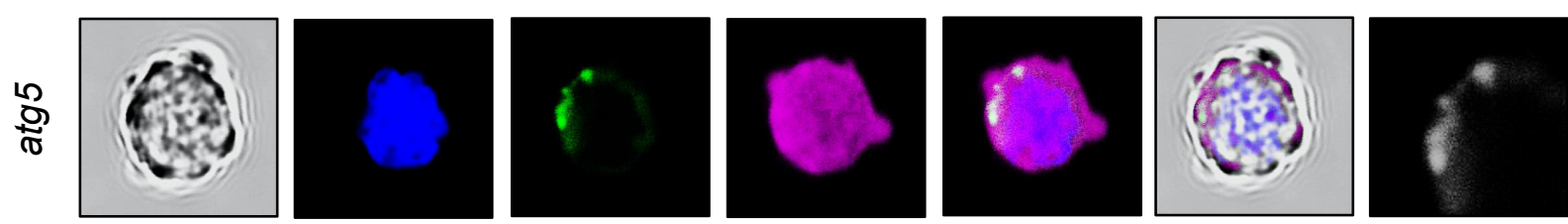

$00_{0}^{0}$
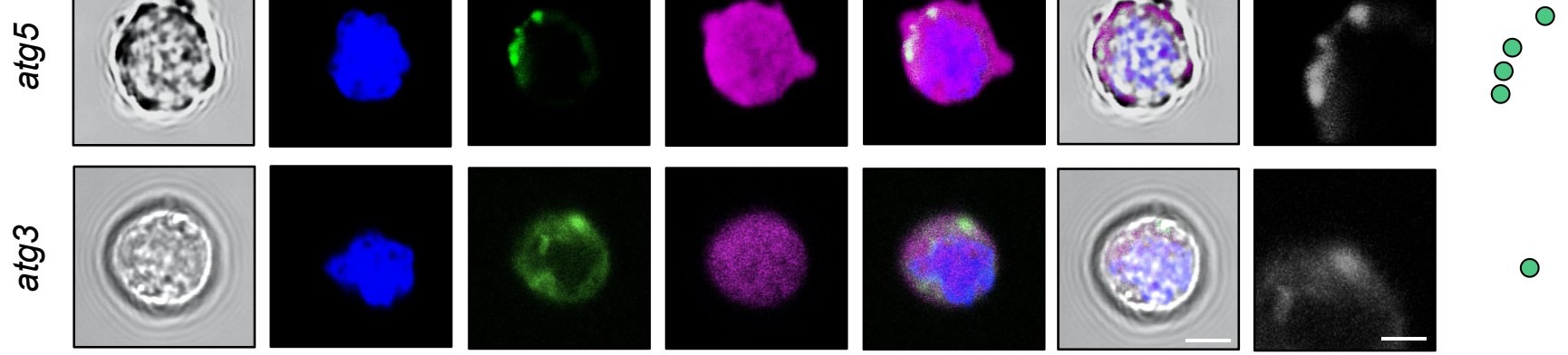

0 
$A$
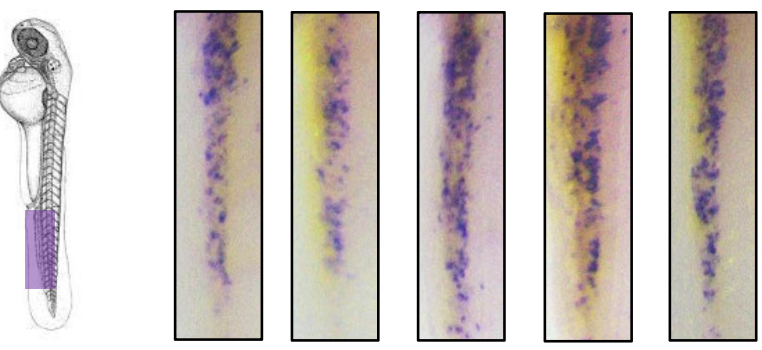

cmyb E

D

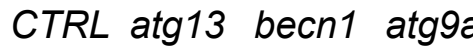

atg2a
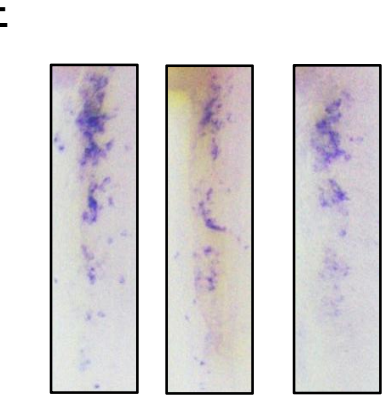

spi1b

G $\mathrm{H}$

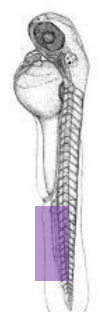

Icp1 J

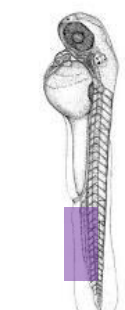

hbae1
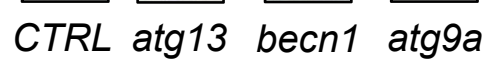
$\mathrm{K}$
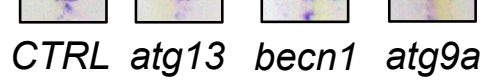

$\operatorname{atg} 2 a$

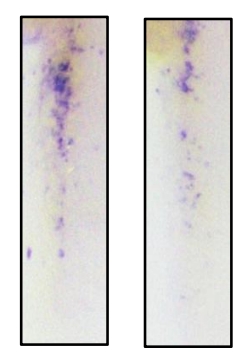

atg2a

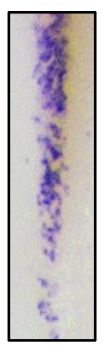

$\operatorname{atg} 5 \operatorname{atg} 3$

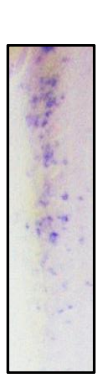

$\operatorname{atg} 5$

$\operatorname{atg} 3$
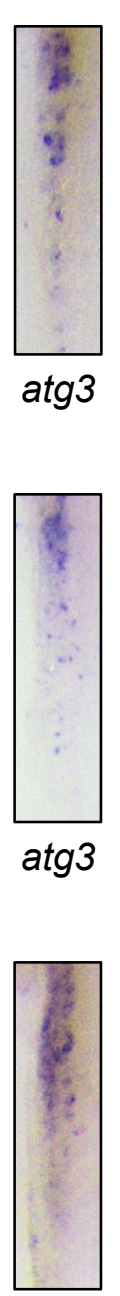

$\operatorname{atg} 3$

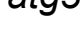

atgs

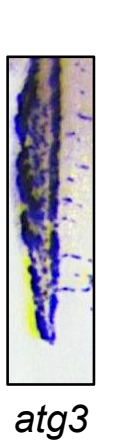

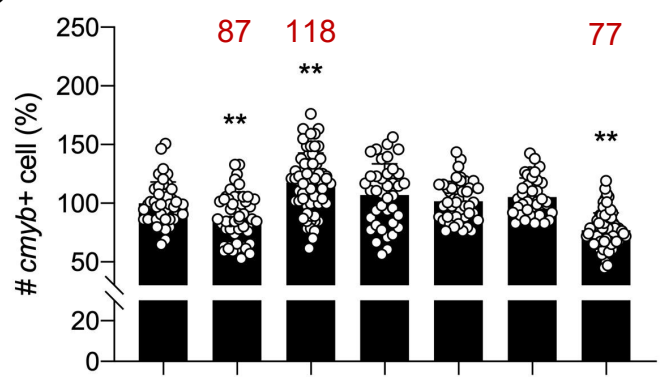

F

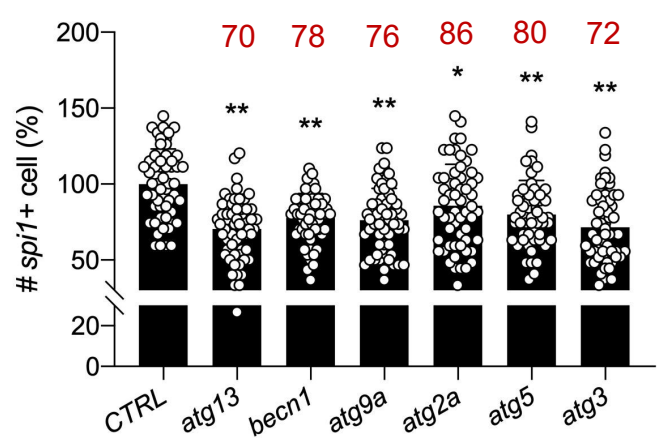

I

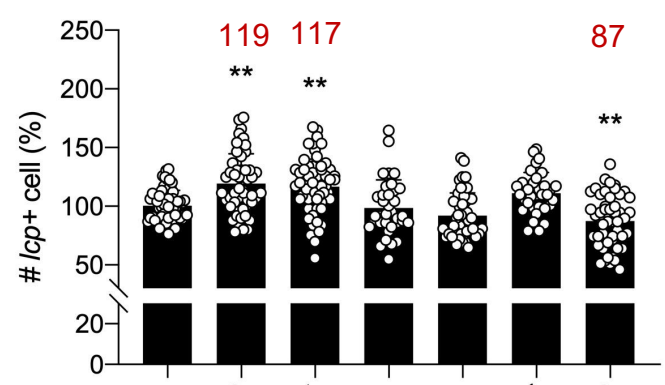

L

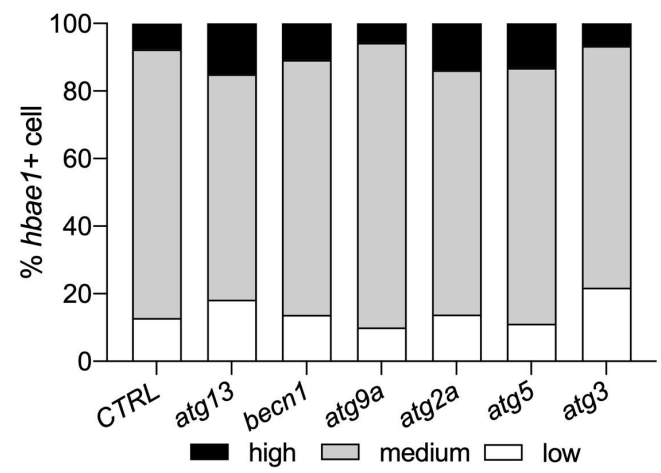




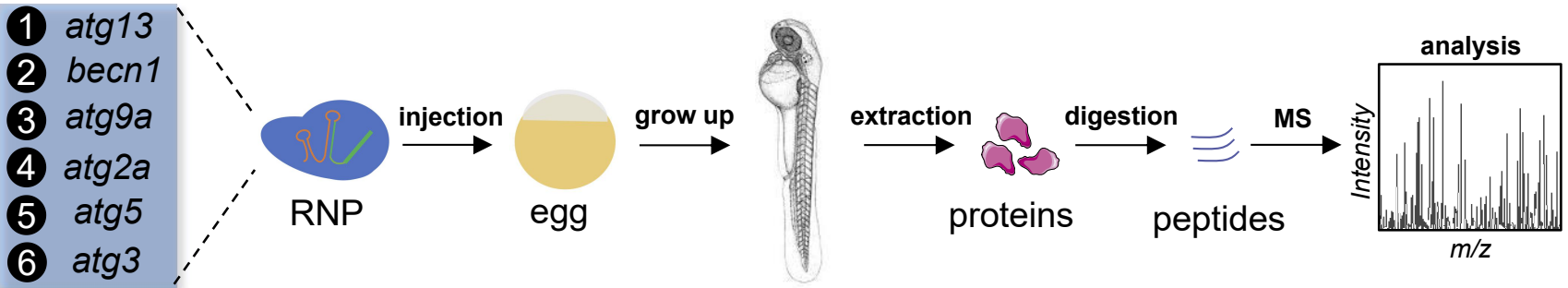

B
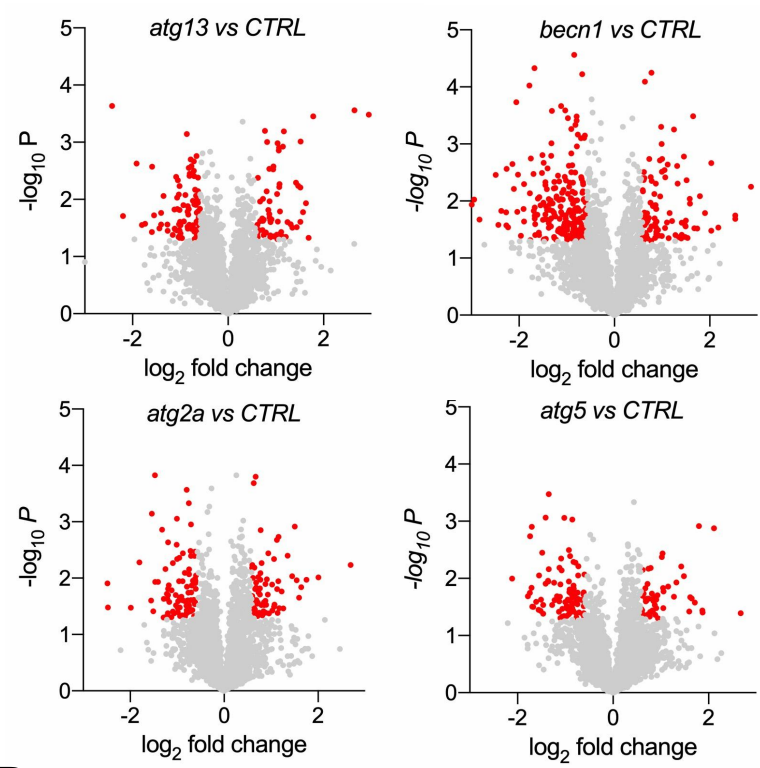

D

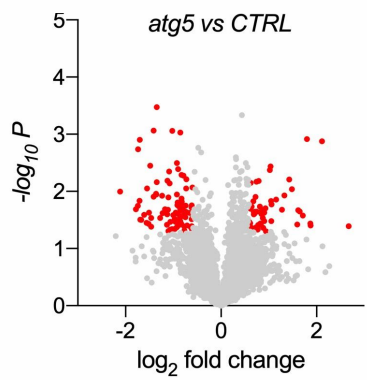

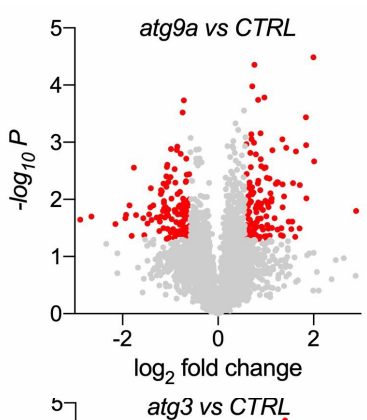

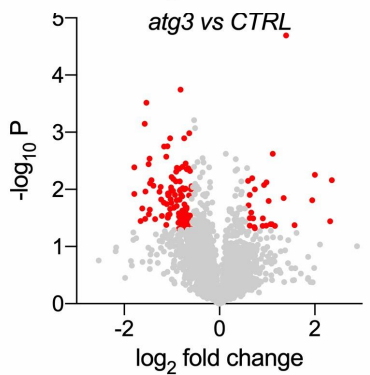

C

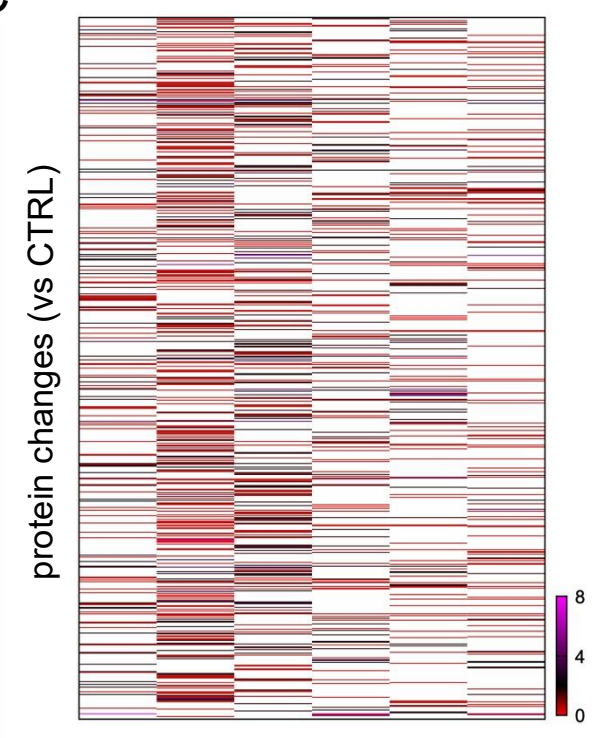

E

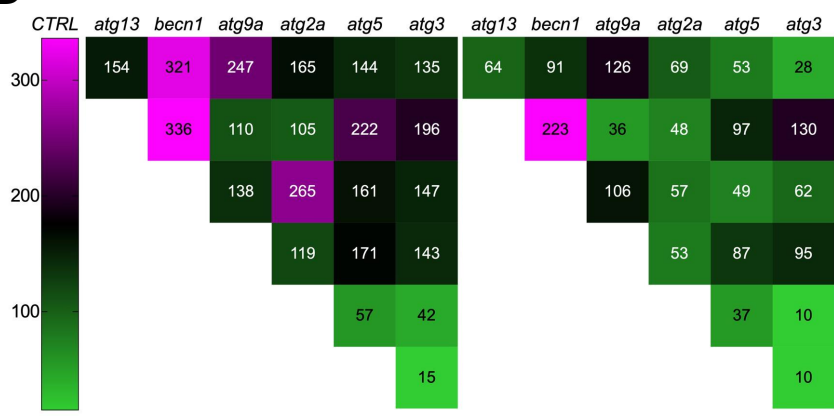

\# changed proteins

F

KEGG pathways

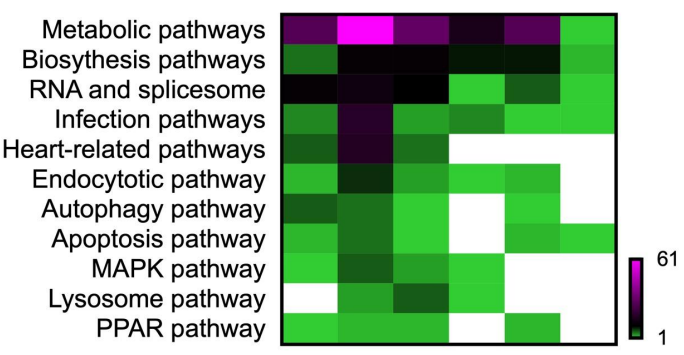

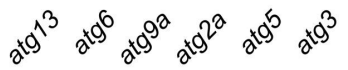

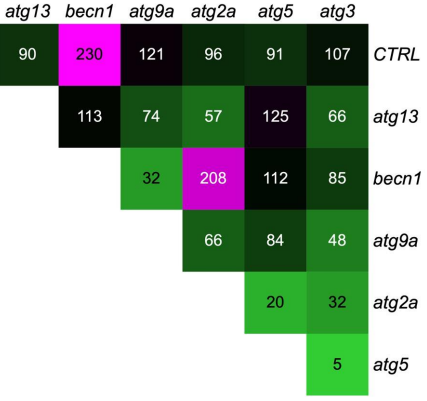

\# decreased proteins

G

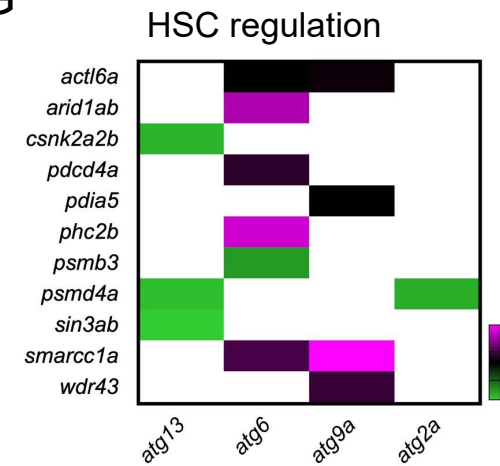

$\mathrm{H}$

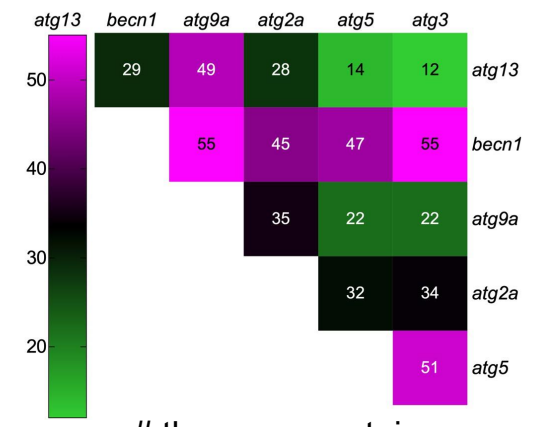

\# the same proteins

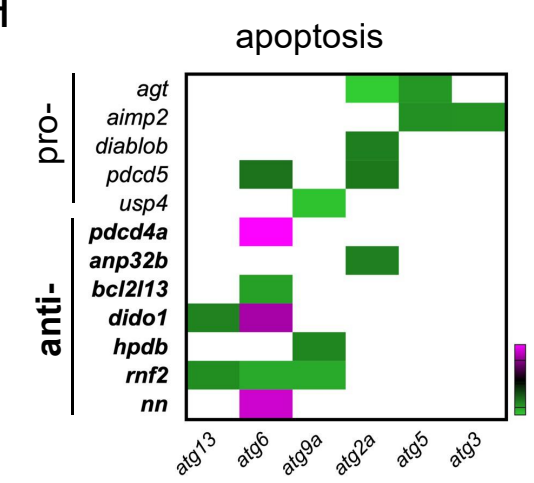




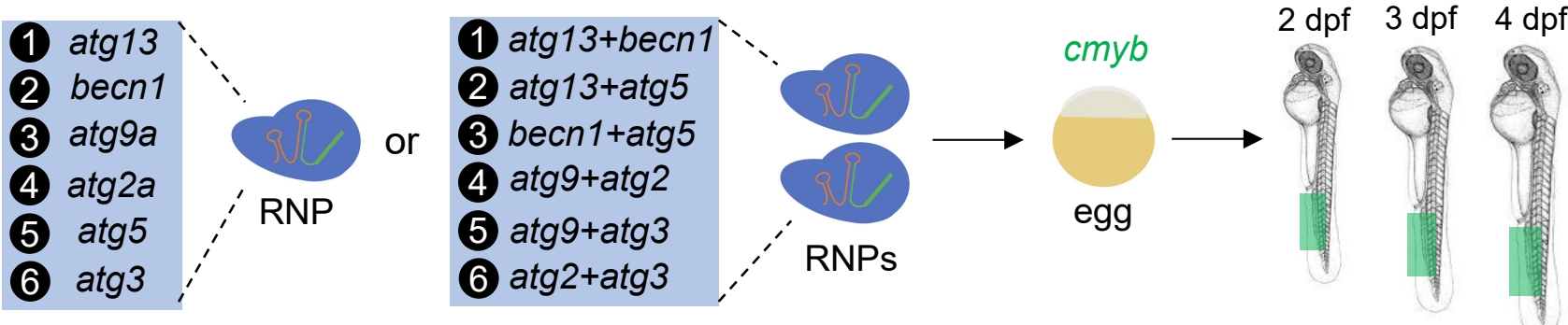

B

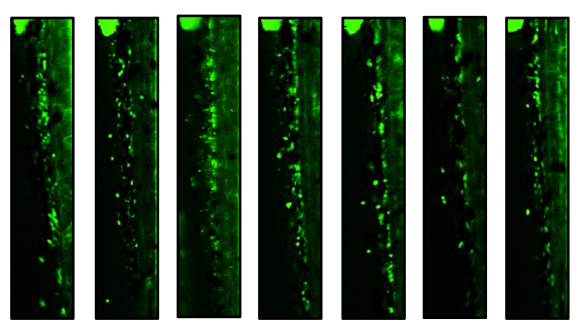

$\begin{array}{lllllll}\text { C } & 13 & \text { b } & 5 & 13 b & 135 & \text { b5 }\end{array}$

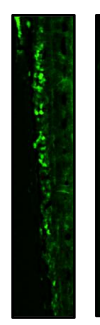

C 13

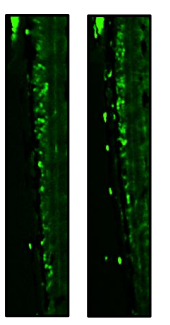

$13 \mathrm{~b}$

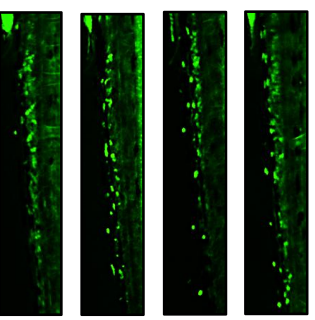

$\begin{array}{llll}5 & 13 b & 135 & b 5\end{array}$
$2 \mathrm{dpf}$

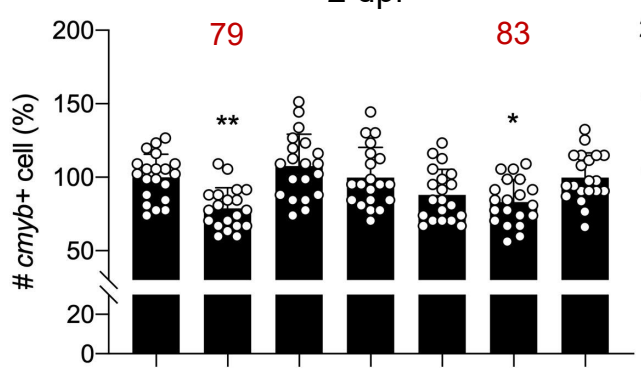

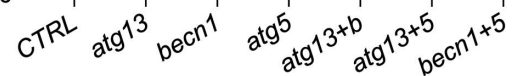

$\mathrm{D}$

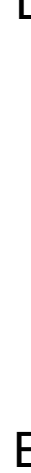

$\mathrm{E}$

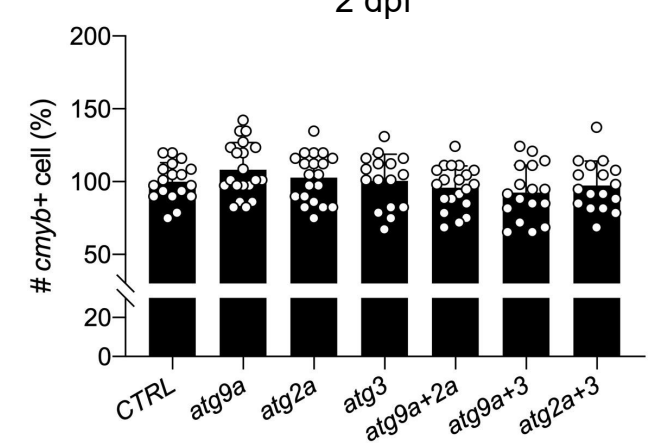

$2 \mathrm{dpf}$

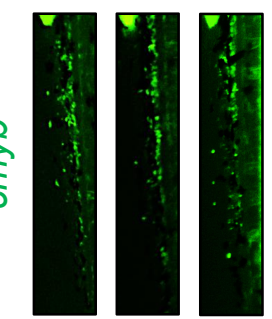

$\begin{array}{lllllll}C & 9 & 2 & 3 & 92 & 93 & 23\end{array}$

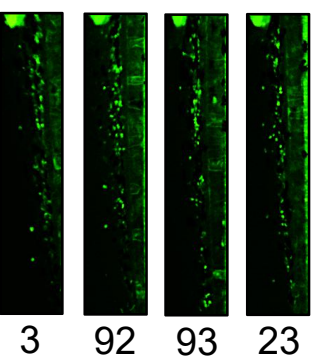

$2 \mathrm{dpf}$
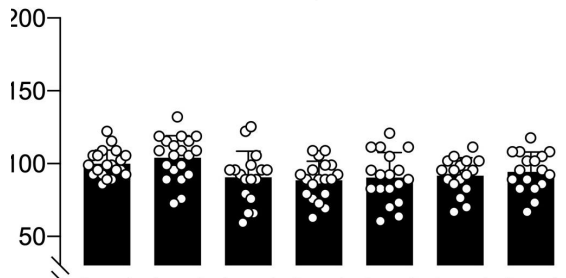

$20-1$

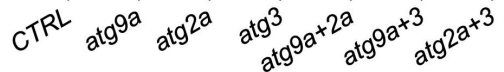

$3 \mathrm{dpf}$

$3 \mathrm{dpf}$
$3 \mathrm{dpf}$

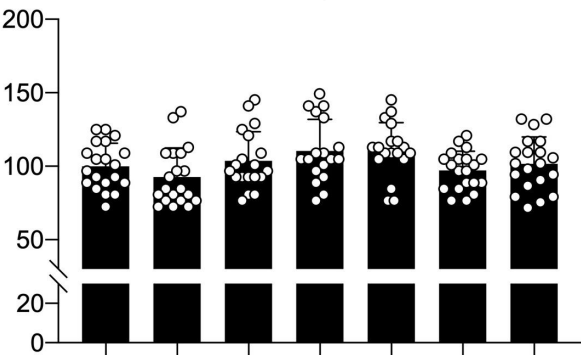

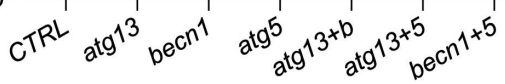
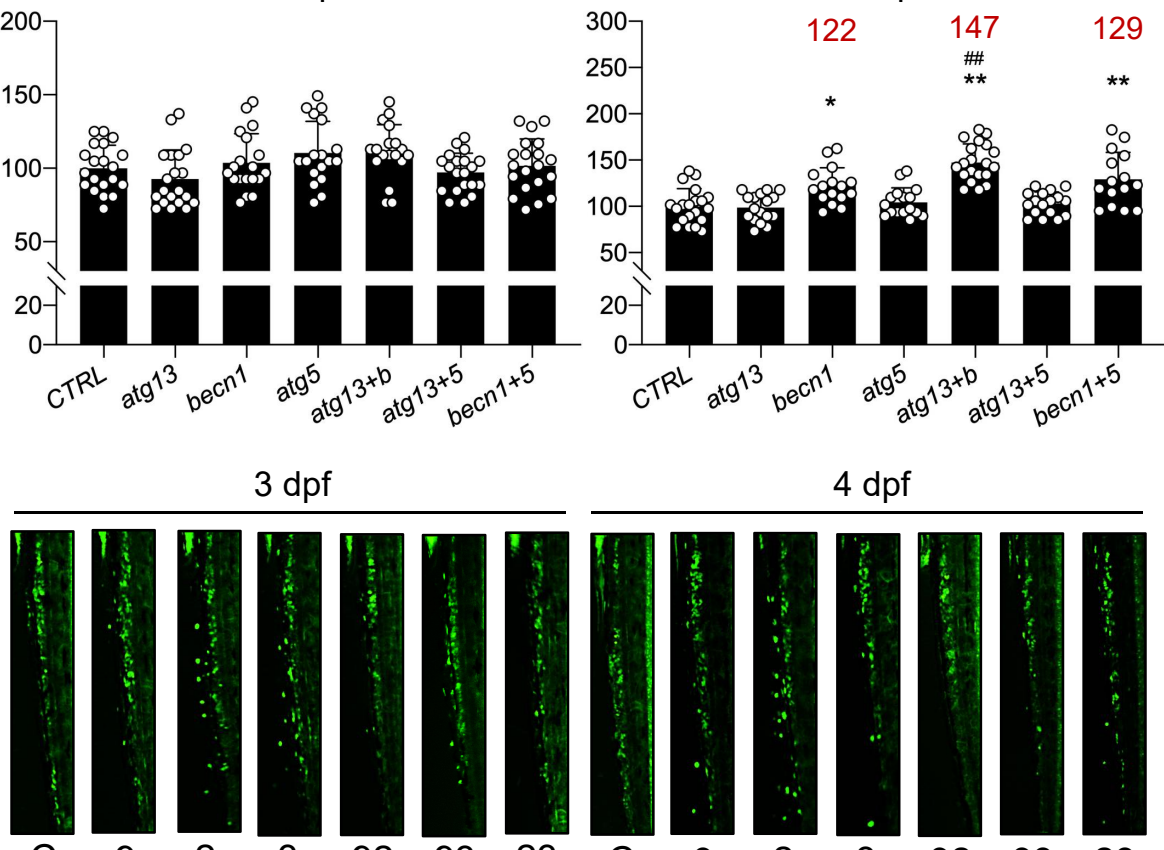

$4 \mathrm{dpf}$
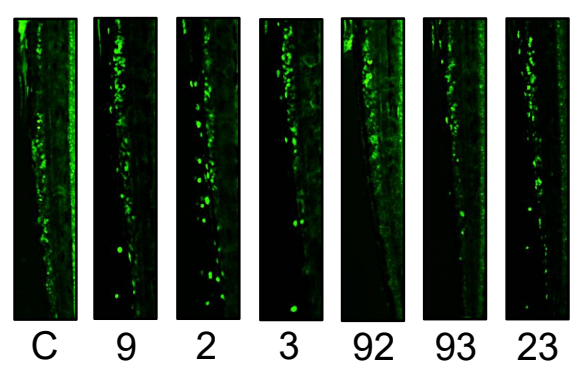

$4 \mathrm{dpf}$
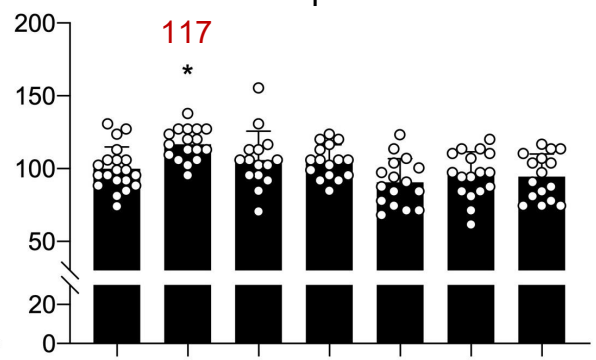

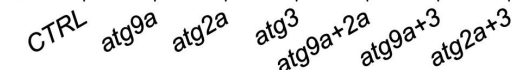



(1) $\operatorname{atg} 13$
2 becn1
(3) $\operatorname{atg} 9 a$
(4) $\operatorname{atg} 2 a$
5 atg5
(6) $\operatorname{atg} 3$
(1) $\operatorname{atg} 13+b e c n 1$ '
or
' RNP
(2) $\operatorname{atg} 13+\operatorname{atg} 5$
(3) becn $1+$ atg 5
(4) $\operatorname{atg} 9+\operatorname{atg} 2$
(5) $\operatorname{atg} 9+\operatorname{atg} 3$
6 atg $2+\operatorname{atg} 3$

B

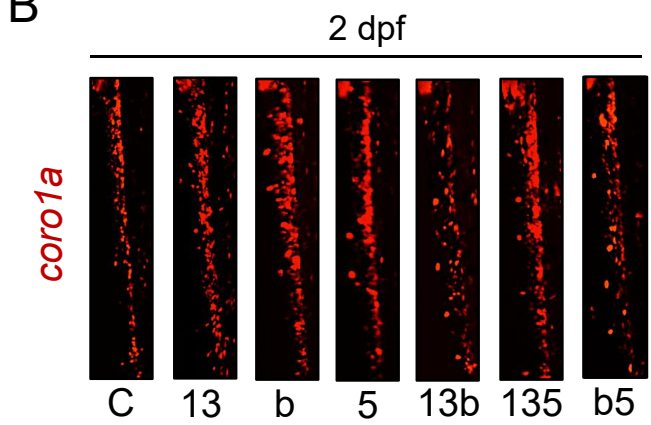

$2 \mathrm{dpf} \quad 3 \mathrm{dpf} \quad 4 \mathrm{dpf}$

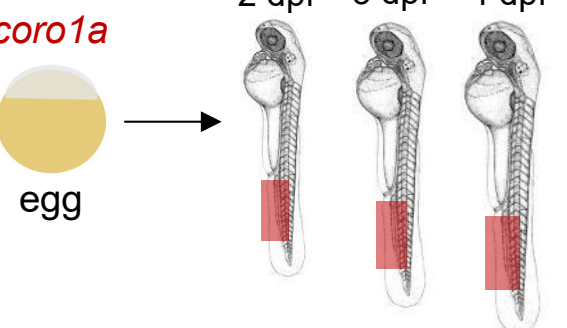

$4 \mathrm{dpf}$

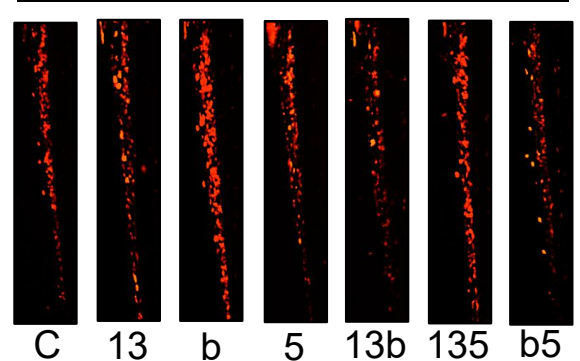

$3 \mathrm{dpf}$

$113 \quad 86 \quad 111$
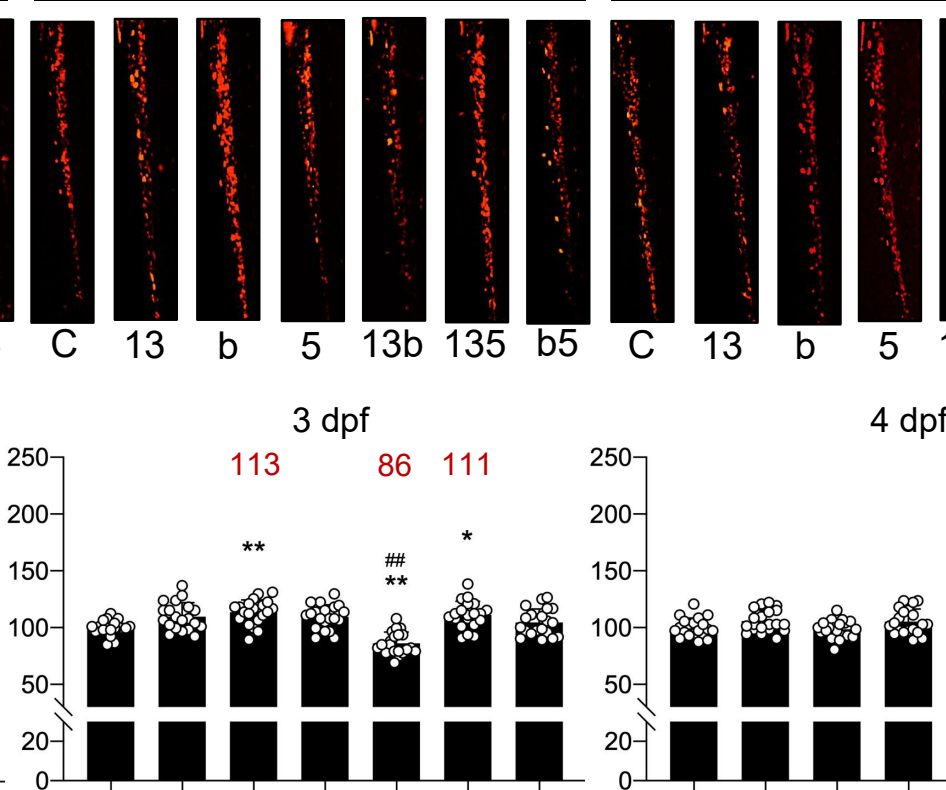

$4 \mathrm{dpf}$
5 13b 135 b5

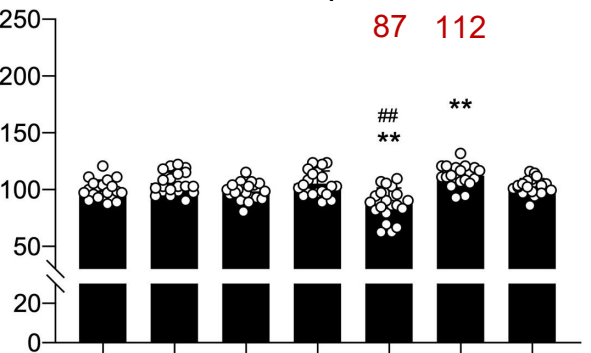

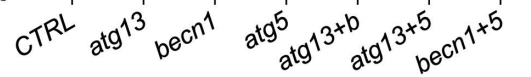

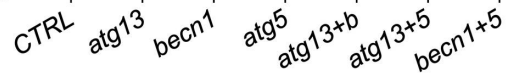

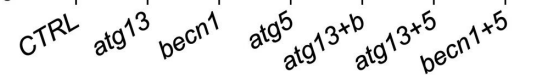

$3 \mathrm{dpf}$

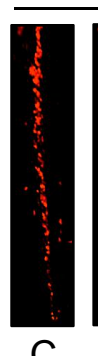

392
$4 \mathrm{dpf}$

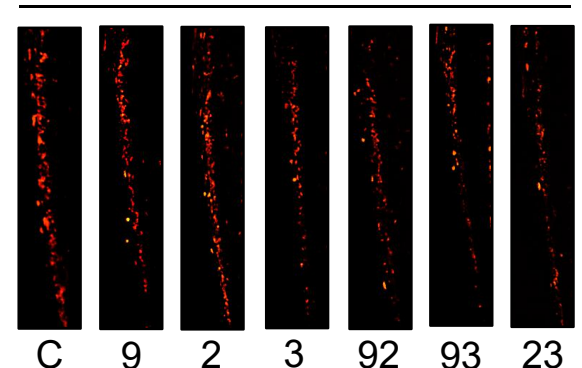

$\begin{array}{lllllll}\text { C } & 9 & 2 & 3 & 92 & 93 & 23\end{array}$

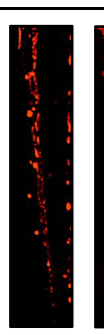

$3 \mathrm{dpf}$

$\begin{array}{lllll}82 & 86 & 89 & 82 & 200\end{array}$

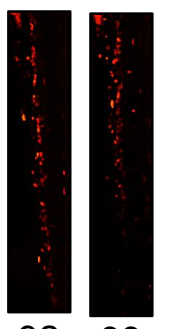

9323

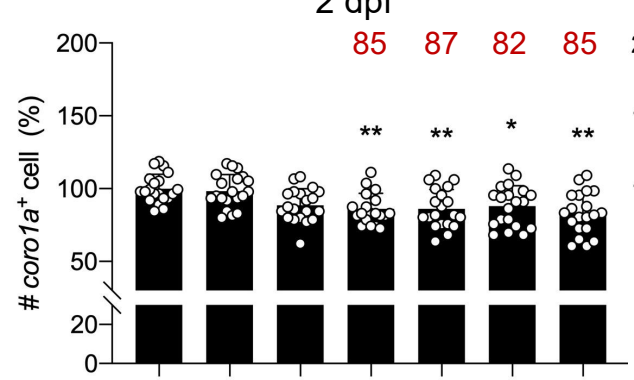

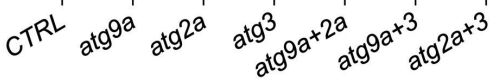

\footnotetext{
200
}
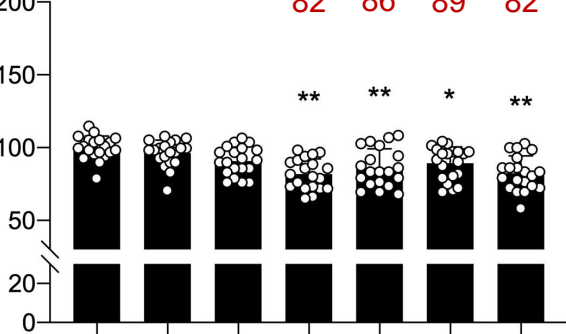

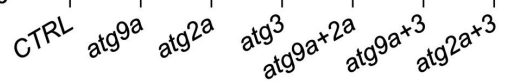
$150-$

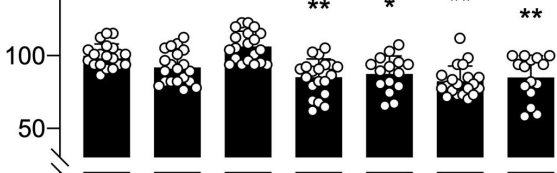
20

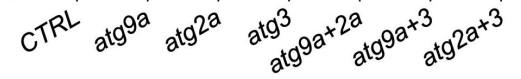


A

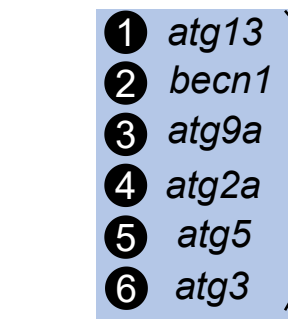

B

B

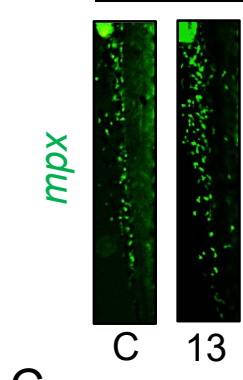

$2 \mathrm{dpf}$

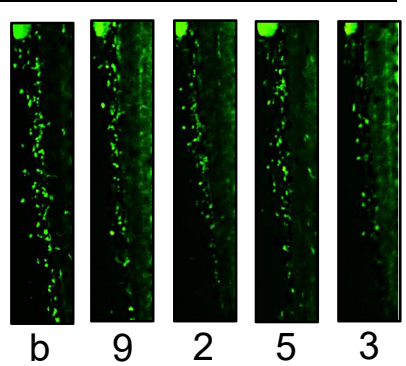

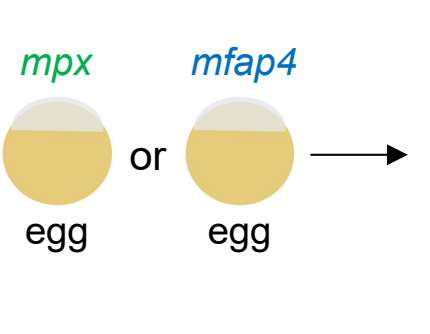

$3 \mathrm{dpf}$
$2 \mathrm{dpf} \quad 3 \mathrm{dpf} \quad 4 \mathrm{dpf} \quad 2 \mathrm{dpf} \quad 3 \mathrm{dpf} \quad 4 \mathrm{dpf}$

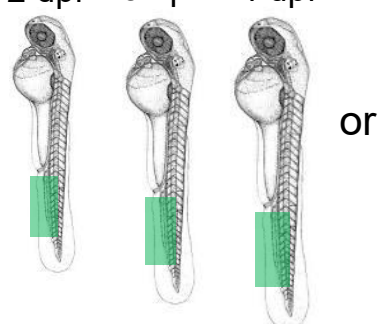

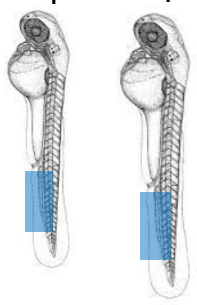

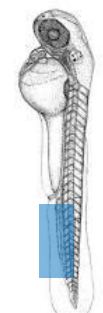

4 dpf

C
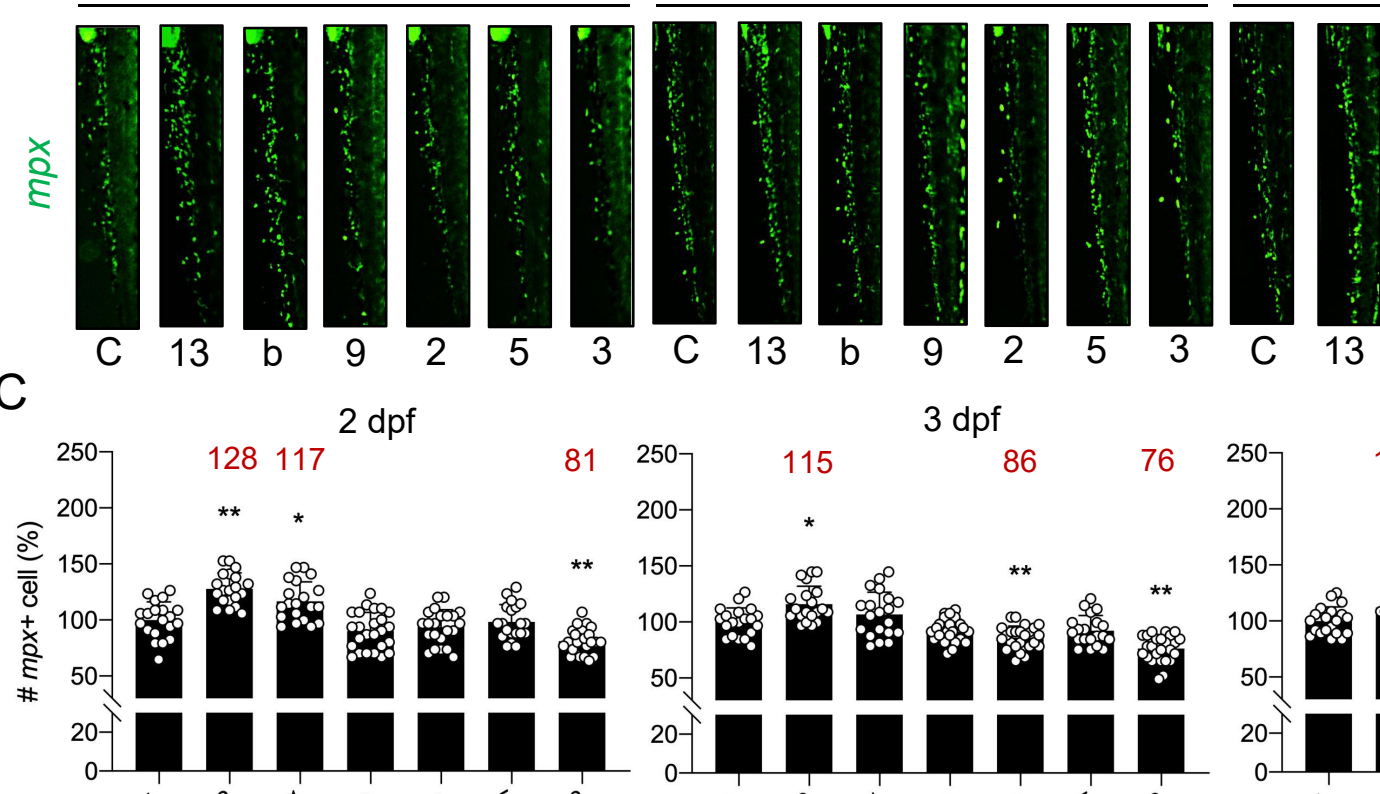

$3 \mathrm{dpf}$
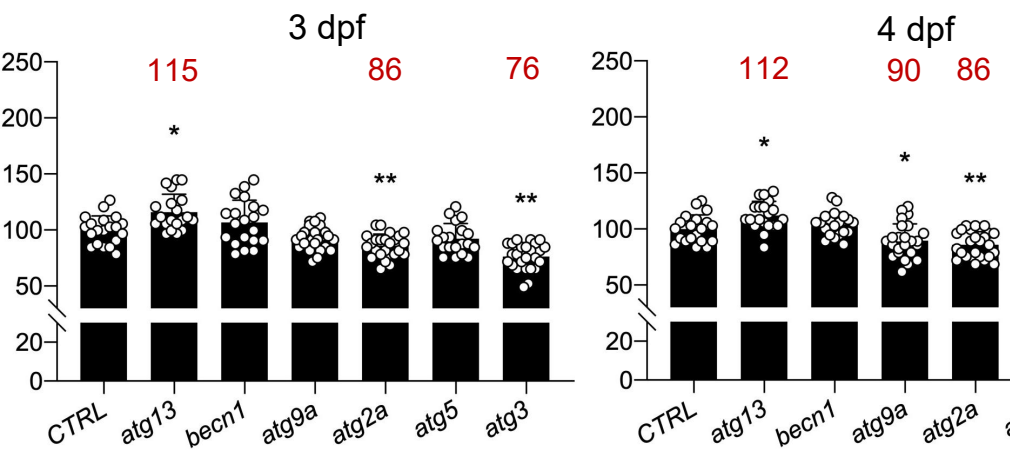

79

D
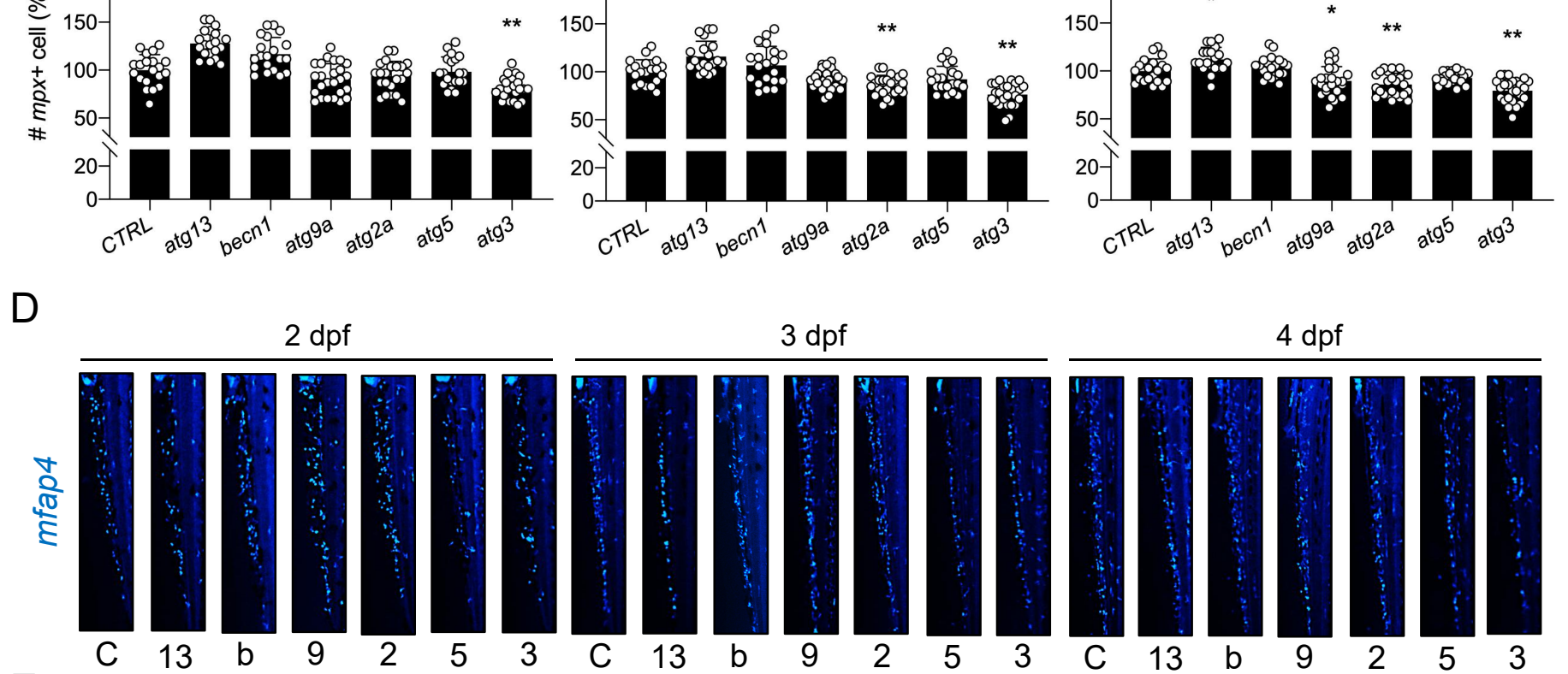

E

$2 \mathrm{dpf}$
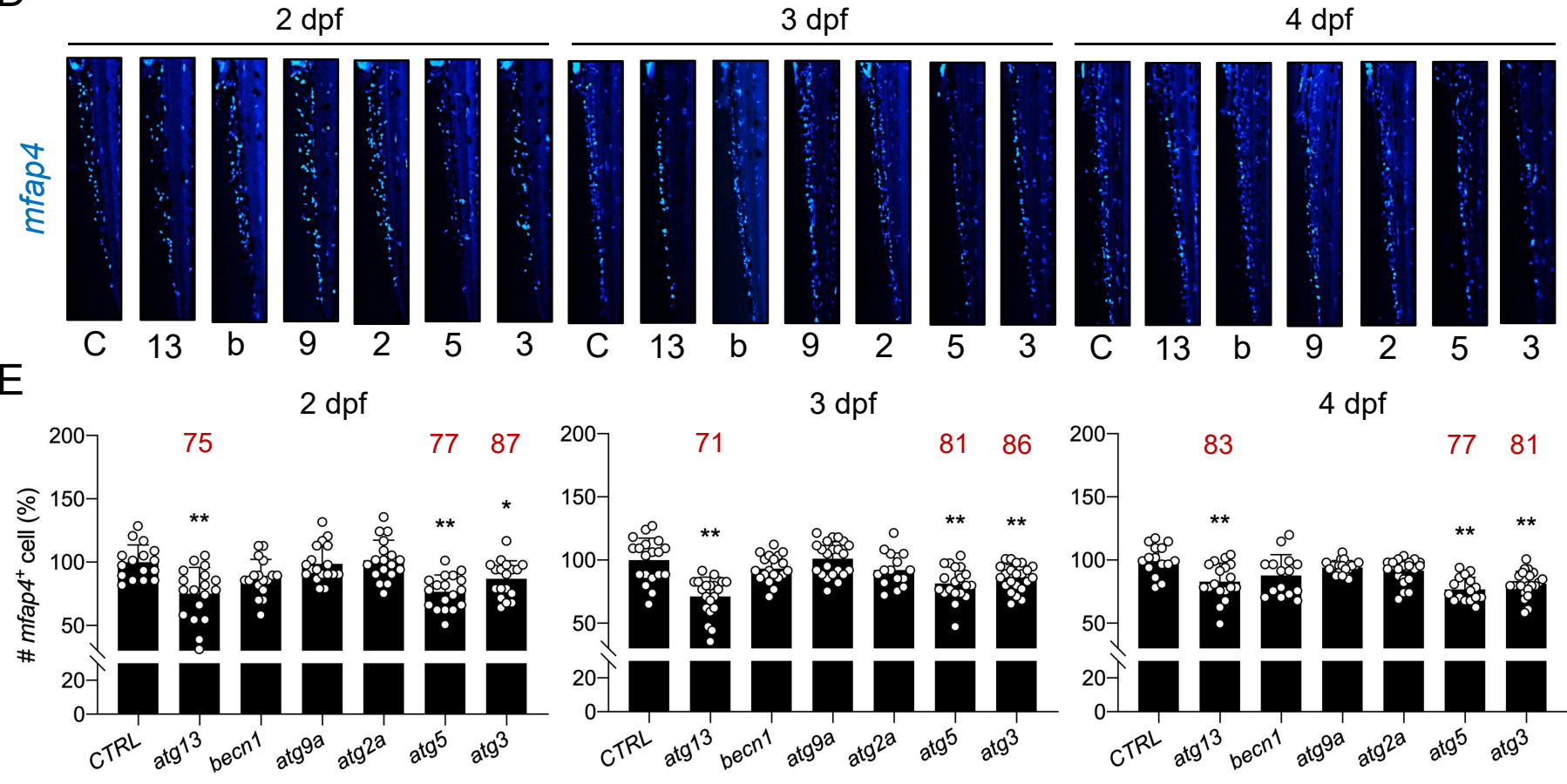

$3 \mathrm{dpf}$

$4 \mathrm{dpf}$

83
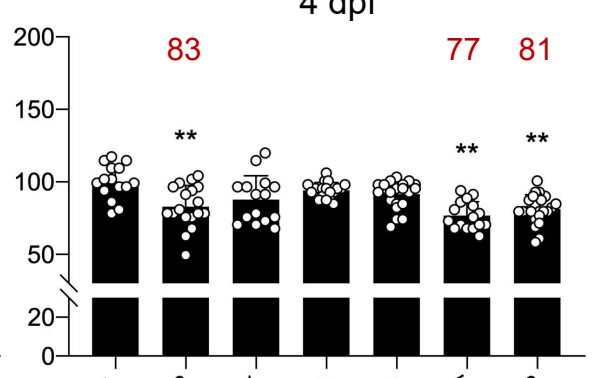

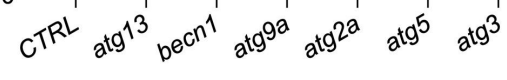

$C^{T R L} a^{t g^{13}} p^{e^{r r^{4}}} a^{t 9^{92}} a^{t g^{22}} a^{t 9^{5}} a^{t 9^{3}}$

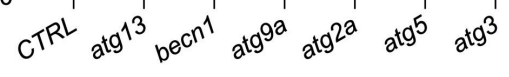

